\title{
Evolution des turbines Kaplan et des groupes axiaux pour l'équipement des installations de fortes puissances et basses chutes
}

\author{
Trends of Kaplan turbines \\ and axial sets for the equipment \\ of high power, low head installations
}

\section{S. Casacci}

Directeur général adjoint Société NeirPic, Grenoble

\author{
J. Bosc
}

Directeur de la Division

Ingénierie Turbines Société Grenoble

\section{Turbines Kaplan}

Les valeurs relatives du débit et de la hauteur de chute ont, pour une puissance donnée une grande influence sur le coût d'un aménagement hydroélectrique. Le prix du kilowatt heure annuel augmente rapidement lorsque les chutes baissent. Les usines de grande puissance furent d'abord pour toutes ces raisons des usines de hautes et moyennes chutes. Mais de tels sites deviennent de moins en moins nombreux et les besoins en énergie exigent la contribution des grandes possibilités des basses chutes.

La turbine Kaplan, avec hélice à pales mobiles, à cause des vitesses spécifiques élevées qu'elle permet d'envisager, s'adapte particulièrement bien à l'équipement des installations de basses chutes. Les développements des moyens de calcul de cette dernière décade apportent, à la conception de ces machines, une aide considérable dans le domaine de la mécanique des fluides et dans l'analyse du comportement dynamique des structures. Ces méthodes deviennent des outils indispensables pour l'étude des turbines Kaplan, de très grandes dimensions, dont les roues peuvent dépasser dix mètres de diamètre. En effet, le dimensionnement des composants de ces énormes machines exige une analyse approfondie des conditions de fonctionnement en marche normale et durant les régimes transitoires.

Les fréquences propres des structures décroissent proportionnellement avec l'augmentation des diamètres. Des phénomènes de résonance, notamment sur les avantdirectrices et les pales de la roue, risquent d'entraîner des fissurations de fatigue pouvant provoquer des dommages appréciables compromettant la bonne tenue de la machine. Pour éviter ces difficultés l'influence de toutes les causes doit être examinée et notamment :

- les phénomènes hydroélastiques (tourbillons de Karman) ;
- les contraintes résiduelles dans les structures soudées; - les concentrations de contraintes sur les avantdirectrices, sur les aubes;

- les défauts admissibles, etc.

Comme application, le présent travail traitera d'un grand aménagement équipé de 18 turbines Kaplan de plus de $100 \mathrm{MW}$ faisant ressortir les caractères spécifiques de ce type de machine.

Installations de basses chutes équipées de turbines Kaplan de grandes dimensions

Pour mettre en évidence les caractères spécifiques de ce type de machine, nous nous appuierons sur l'aménagement de Porto Primavera (Brésil).

Cette installation réalisée par Centrais Eletricas de Sao Paulo sur le rio Parana comporte 18 groupes ayant les caractéristiques nominales suivantes :

$-N=103 \mathrm{MW}$;

$-H=18 \mathrm{~m}$;

$-n=75 \mathrm{tr} / \mathrm{min}$.

La mise en service du premier groupe est prévue en 1986.

La figure 1 montre la disposition générale de la machine. Le groupe complet comporte 3 paliers : deux paliers, de part et d'autre de l'altematuer, s'appuyant sur leur croisillon respectif et un palier au niveau de la turbine. Les charges axiales sont reprises par le pivot reposant sur le fond de la turbine; la transmission des charges, sur les patins, se fait au moyen :

- d'un arbre intermédiaire disposé entre le rotor de l'alternateur et l'arbre turbine ;

- et d'un manchon.

Le flasque supérieur de la turbine est coupé en deux parties concentriques pour permettre le démontage de 


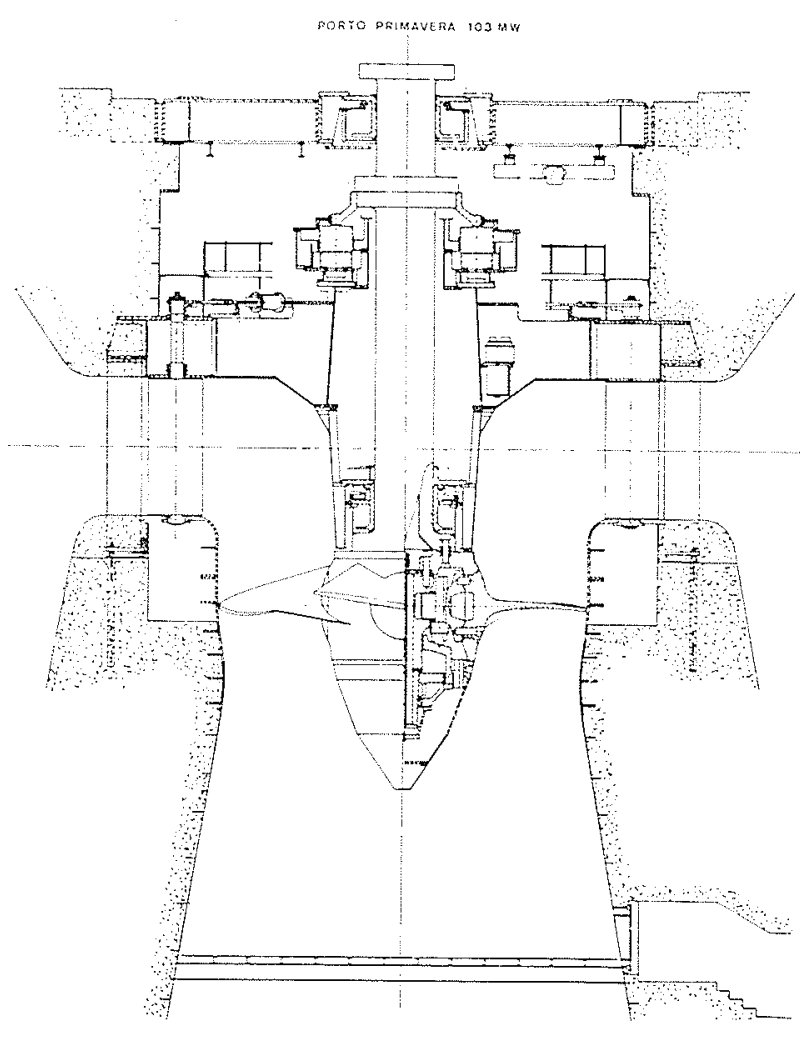

Figure 1. - Centrale de Porto Primavera (Brésil) - Coupe de la turbine Kaplan de $103 \mathrm{MW}$

la roue sans démonter le distributeur. La partie centrale, de structure caissonnée très rigide, supporte à sa partie inférieure, le palier turbine et à sa partie supérieure, le pivot. Pour des raisons de rigidité maximale, la partie extérieure, qui comporte les directrices, est bétonnée après sa fixation sur les avant-directrices. Cette disposition supprime une bride supplémentaire entre le béton et la partie portant les directrices, ce qui évite une flexibilité et un joint d'étanchéité et réduit l'encombrement. Par contre, le démontage des directrices doit s'effectuer par le bas, dans une chambre annulaire autour de la ceinture de roue.

La commande du distributeur se fait par le moyen d'un cercle de vannage entraîné par quatre servo-moteurs attachés sur le flasque support de pivot.

La roue comporte quatre pales rapportées en acier inoxydable, mues par un servo-moteur logé dans la roue sous les pales et dont l'alimentation est assurée au moyen de tubes dans l'arbre et d'alvéoles de distribution reposant sur le croisillon supérieur de l'alternateur.

Quatre soupapes d'entrée d'air, disposées dans la partie centrale du flasque supérieur, permettent de limiter la dépression sous la roue au cours des fonctionnements transitoires de la machine.

Après cette rapide description, nous allons développer quelques points essentiels de la conception des groupes qui se rapportent :

- à la roue ;

- à l'avant-distributeur ;

- au pivot;

- au système de réglage.

\section{Etude et conception des roues}

Elle comporte quatre pales de $8,6 \mathrm{~m}$ de diamètre.

Le dimensionnement de cet organe fait appel aux derniers développements de l'informatique dans les domaines de la mécanique des fluides et de la mécanique des structures.

Le calcul des tracés hydrauliques s'effectue à l'aide de programmes quasi-tridimensionnels qui prennent en compte les caractéristiques spécifiques de la machine étudiée. Nous réservons les essais sur modèle d'une part, à l'optimisation très fine exigée par le haut niveau des performances, d'autre part au contrôle final des résultats concernant les rendements, le fonctionnement hors des zones de meilleurs rendements, ainsi que le comportement de la machine aux phénomènes de cavitation, de stabilité, aux régimes transitoires, etc.

Depuis près de 15 ans, nous avons perfectionné notre méthode de calcul des tracés. Elle consiste à modéliser mathématiquement, en première approximation, les conduits hydrauliques par un ensemble de filets fluides en équilibre radial. Ces premières données permettent de calculer l'écoulement dans la machine élémentaire constituée par la grille d'aubes tracée sur les surfaces de révolution caractérisant le filet fluide correspondant. Ces résultats servent, ensuite, à définir une nouvelle approximation du réseau d'écoulement méridien jusqu'à convergence de la solution. Par itération on obtient donc une bonne représentation de l'écoulement dans les organes d'alimentation, le rotor et l'évacuation de la turbomachine.

Cette méthode fait appel à deux programmes couplés :

- calcul de l'écoulement, en général rotationnel, dans les conduits axisymétriques, l'effet d'une grille d'aubes est représenté par un champ de forces judicieusement choisi ;

- calcul de l'écoulement dans une grille d'aubes tracée sur une surface de révolution de méridienne quelconque.

Ces deux programmes utilisent avantageusement les mêmes types d'algorithmes qui permettent de transformer des équations du type Poisson (le second membre comportant des fonctions inconnues non linéaires) en équations intégrales de Fredhom de deuxième espèce.

La mise au point de ces programmes exigeait une bonne convergence n'entrainant pas un volume de calculs trop important qui en aurait limité l'application. Actuellement, le nombre de points "calcul" a été considérablement réduit par l'application de nouvelles méthodes numériques développées par notre équipe de spécialistes.

Dans la pratique, on conduit le calcul en considérant les formes exactes des pièces constituant les canaux hydrauliques (voir Fig. 2) : ceinture, plafond, moyeu, pointe de roue. On traite l'ensemble de l'écoulement à partir de l'entrée du distributeur et jusqu'à la sortie du cône droit de l'aspirateur. Le distributeur impose l'écoulement à l'entrée de la roue.

Le programme fournit :

- les lignes de courant ;

-.. Les répartitions de vitesse et de pression sur les profils le long des lignes de courant (voir Fig. 3). 


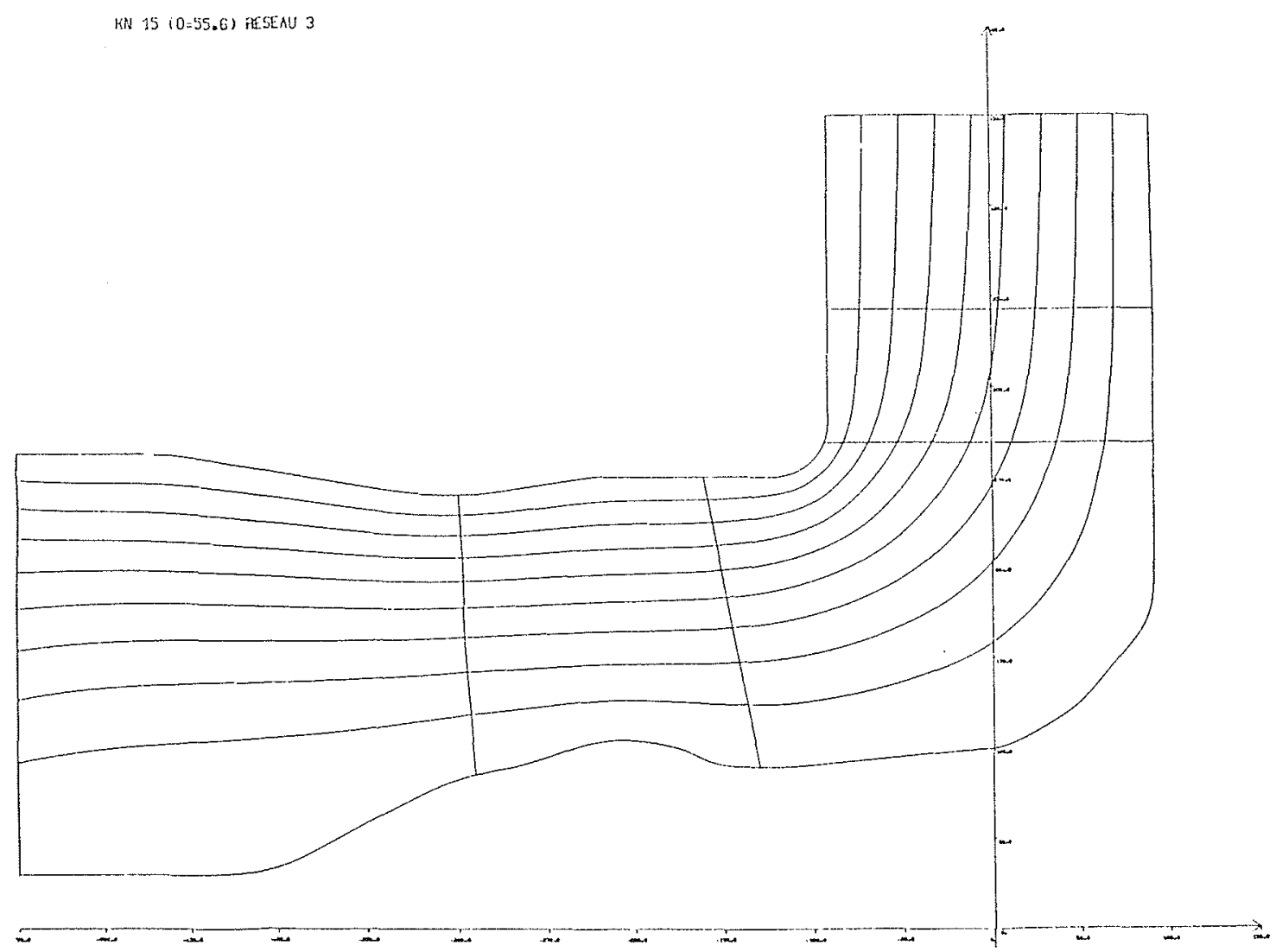

Figure 2. -- Ecoulement méridien dans le conduit hydraulique d'une Kaplan depuis l'entrée du distributeur jusqu'à l'entrée du coude d'aspirateur.

Le lissage des formes géométriques se fait à l'aide d'un programme en interactif.

On peut modifier, si nécessaire, le profil et se fixer une loi d'épaisseur et une loi de cambrure. Le programme de calcul par itérations assure la continuité entre les profils définis pour les différents canaux. La géométrie doit, bien entendu, tenir compte des impératifs de dimensionnement mécanique.

Ce programme présente un grand intérêt pour l'amélioration locale des tracés pour réduire ou supprimer les poches de cavitation. Il peut permettre de modificr unc rouc industrielle, sujette à des usures par cavitation, ayant pour origine des erreurs de définition des chutes de fonctionnement. L'ensemble des programmes conduit à la définition informatisée des tracés et fournit toutes les données nécessaires aux calculs mécaniques de la roue :

- masse, centre de gravité ;

- sections, inerties;

- inertie massique ;

- efforts centrifuges (forces et moments).

La connaissance des pressions le long des profils, qui entraîne celle des efforts hydrauliques (couples, poussée axiale), rend possible le calcul de la pale par la méthode des éléments finis.

La forme générale des pales Kaplan, coques relativement minces, aux variations d'épaisseur très progressives, permet l'utilisation d'éléments de plaques d'épais- seur uniforme pour une étude des comportements statique et dynamique.

Pour la modélisation des pales Kaplan, nous utilisons un programme spécial de maillage automatique mis au point pour les aubes des roues Francis.

La connaissance plus précise de la distribution des contraintes à l'attache de la pale sur le plateau, au droit des rayons de raccordement, exige une étude générale sur les liaisons des éléments de coque avec des éléments massifs tridimensionnels dans un programme de calcul par éléments finis.

A titre d'exemple de calcul des pales, au stade du projet, nous donnons quelques résultats relatifs à une turbine Kaplan, d'une puissance maximale de $156 \mathrm{MW}$, sous une chute de $24,1 \mathrm{~m}$; diamètre de roue $=$ $9600 \mathrm{~mm}$ (fig. 4 et 5).

Nous déterminons également les modes et fréquences propres de la pale, nécessaires pour l'étude du comportement dynamique en fonctionnement. Les essais industriels sur certaines de nos machines montrent que les perturbations créées par une dissymétrie d'écoulement à l'amont de la roue, dues à une mauvaise conjugaison, à des décollements sur les pales, ... peuvent entraîner des résonances qui accroissent les efforts pulsatoires sur les pales. Certains mouvements vibratoires de la pale, relatifs aux modes propres, peuvent être excités par des harmoniques de la fréquence de passage des pales, même de rang élevé. Ajoutées à la valeur moyenne 


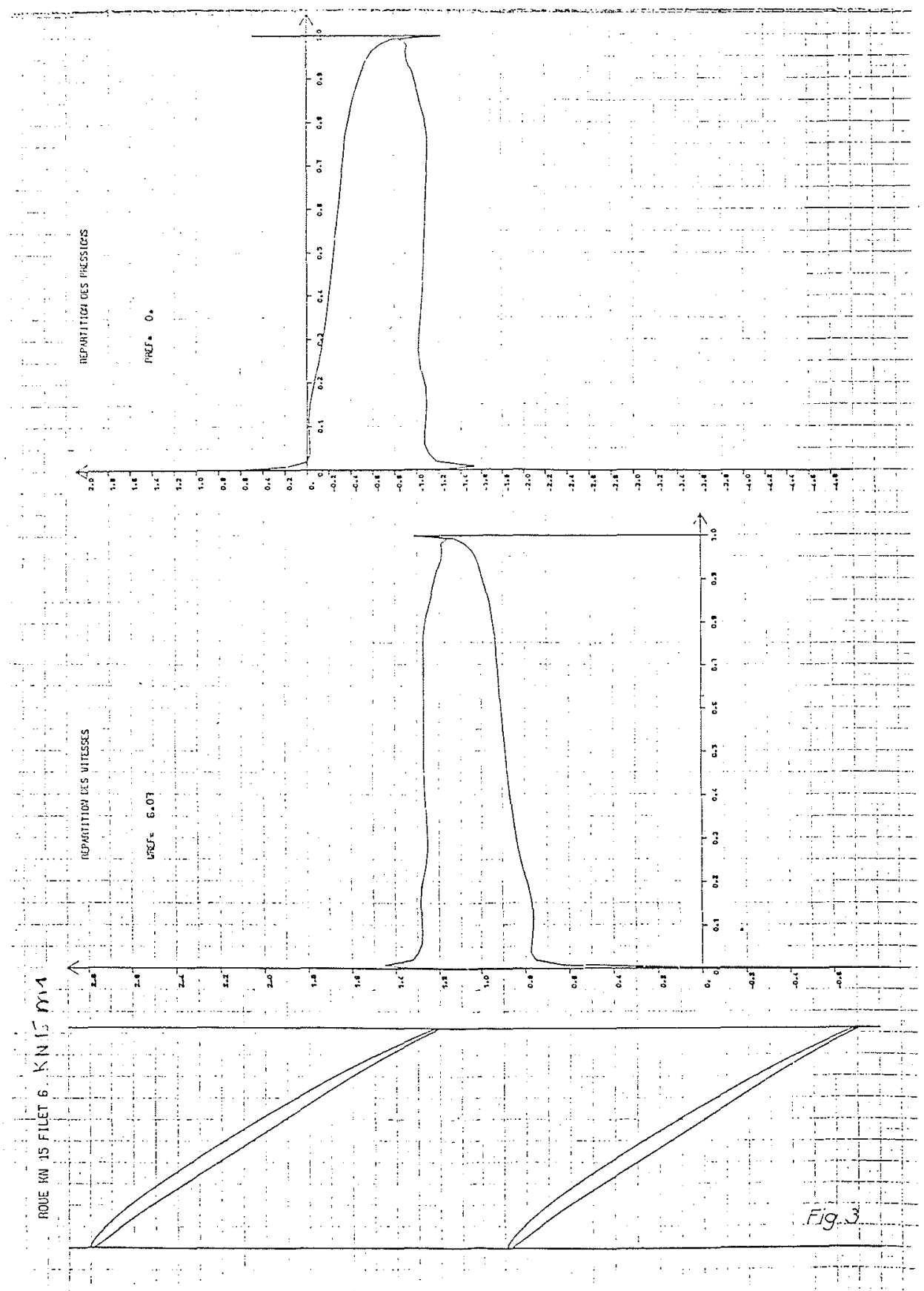

Figure 3. - Exemples de répartition de vitesse et de pression sur les profils.

des contraintes, les composantes à fréquences élevées permettent de définir la tenue à la fatigue des pales.

Le moyeu, élément important de la roue, constitue une pièce massive dont les formes et les épaisseurs rendent difficile l'analyse de la répartition des contraintes. L'étude du dimensionnement, conduite selon une schématisation de la pièce, à partir d'éléments tels que plaques, anneaux, poutres à inertie variable, devient insuffisante. Des programmes de calculs par éléments finis, à partir d'éléments massifs tridimensionnels à 6 et 8 nœuds, permettent une modélisation beaucoup plus représentative. La figure 6 donne un exemple du maillage pour un quart de moyeu relatif à l'affaire déjà citée.
Cette méthode permet l'optimisation du moyeu par la meilleure connaissance de la distribution des contraintes dans les différentes parties de la pièce. Une telle étude approfondie nous a permis de choisir, pour les moyeux des machines de Porto Primavera, une nuance d'acier de caractéristiques mécaniques conventionnelles. L'élaboration, beaucoup plus aisée en fonderie de ce type d'acier, conduit à des pièces de bonne qualité, de surcroît plus faciles à réparer.

\section{Avant-distributeur}

L'avant-distributeur des turbines de Porto Primavera comporte 12 avant-directrices uniformément réparties 


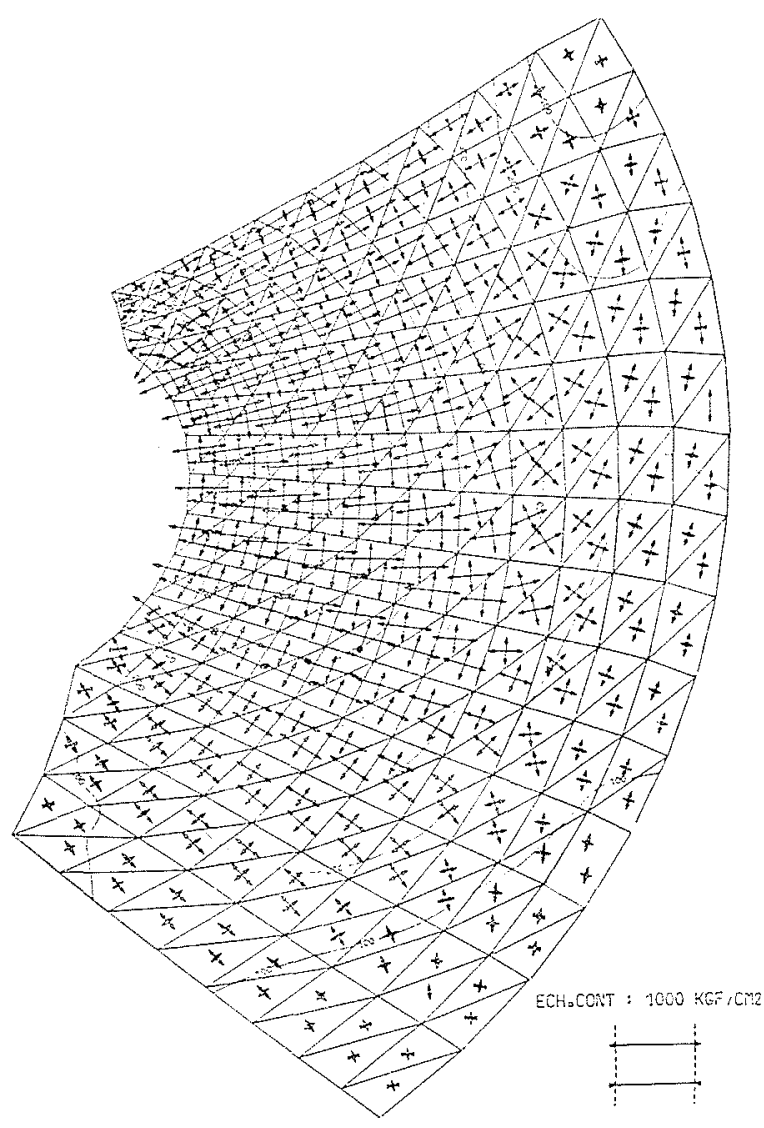

Figure 4. - Yacireta Apipe - Distribution des contraintes sur l'intrados pour un fonctionnement à la puissance maximale.

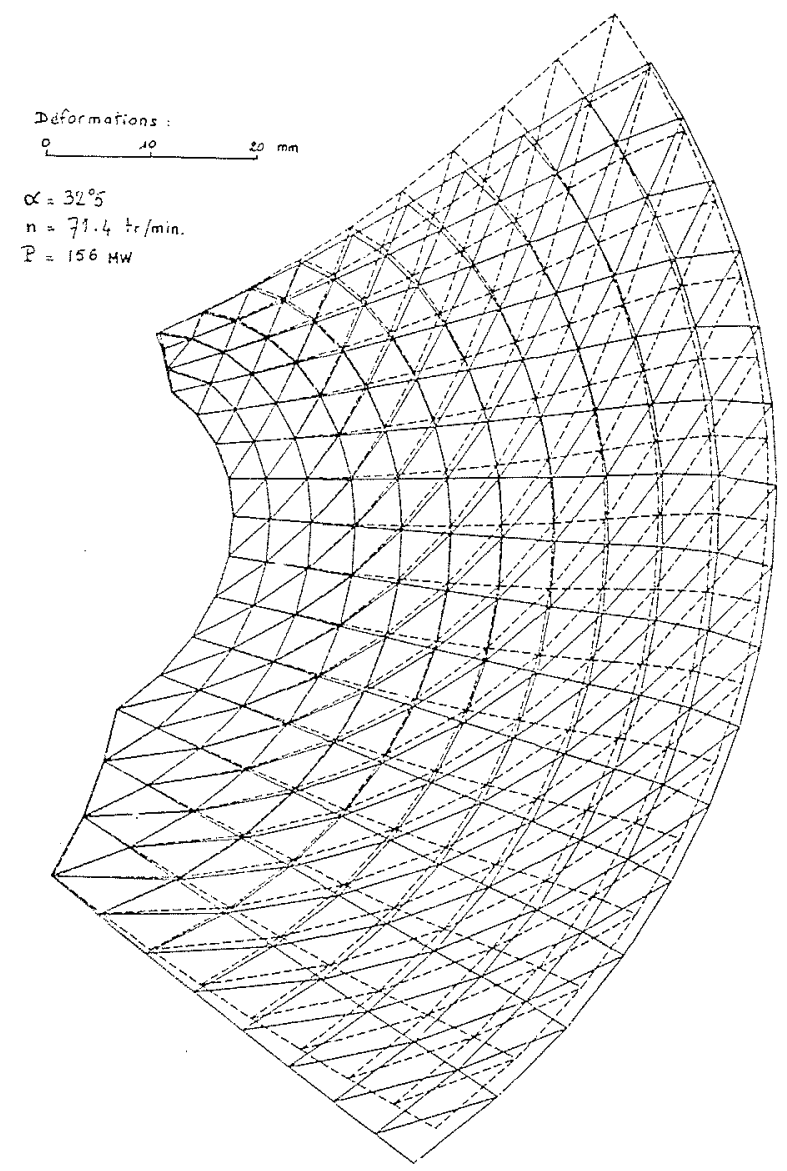

Figure 5. - Yacireta Apipe - Déformation de la pale vue de dessus pour le même fonctionnement.

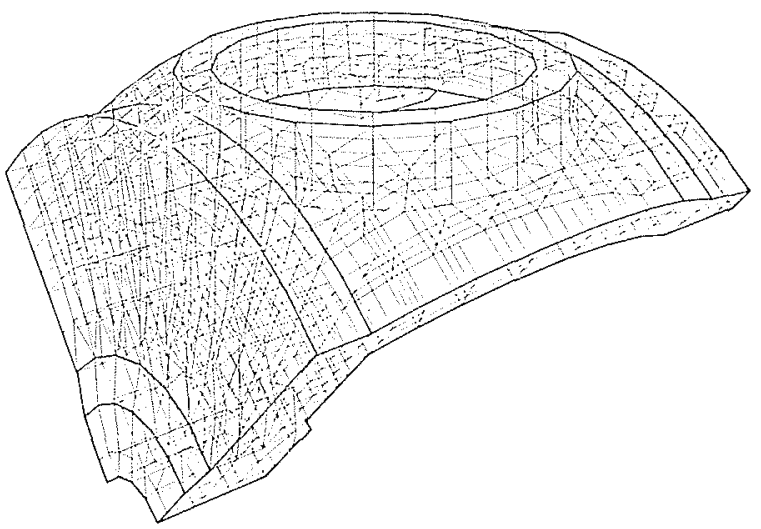

Figure 6. - Yacireta - Corps de moyeu - Perspective du maillage.

sur le pourtour et 3 avant-directrices supplémentaires pour assurer un meilleur guidage de lécoulement dans la partie frontale de la bâche du côté du bec.

Compte tenu des dimensions importantes de cette pièce : diamètre extérieur des avant-directrices : $13,7 \mathrm{~m}$ environ et hauteur du distributeur : $3,2 \mathrm{~m}$ environ, nous avons choisi des avant-directrices creuses. Cette disposition permet l'obtention de meilleures formes hydrauliques qui réduisent des forces excitatrices pulsatoires liées, soit à des tourbillons de Karman sur l'arête de sortie, soit à des décollements sur l'arête d'entrée. Ces excitations peuvent exister, soit en régime permanent pour certaines charges, soit momentanément au cours des fonctionnements transitoires : les avant-directrices vibrent à leur fréquence propre dans l'eau, les amplitudes de contraintes varient selon les régimes de fonctionnement.

Conjointement à la réduction des efforts hydrauliques pulsatoires, l'adoption d'avant-directrices creuses conduit à des fréquences propres plus élevées : les deux facteurs vont donc dans le sens d'un meilleur comportement des avant-directrices aux excitations liées à l'écoulement.

\section{Pivot - Conception et comportement thermo- hydrodynamique}

Ce qui fait la difficulté particulière des pivots des grandes Kaplan consiste en leur charge élevée appliquée sur un petit diamètre moyen. Il en résulte un faible nombre de patins, de dimensions relativement importantes.

Le pivot de Porto Primavera supporte une charge de 2000 tonnes sur 9 patins; la couronne d'appui a un diamètre moyen de $3025 \mathrm{~mm}$ et une largeur de $750 \mathrm{~mm}$.

Pour améliorer la sécurité des grandes pivoteries, il est indispensable de réaliser une équirépartition automatique des charges. Sur nos pivots, au-delà de 1000 tonnes, celle-ci est réalisée par un ensemble de membranes toriques en acier de faible épaisseur, remplies d'huile et reliées entre elles par un circuit commun, et sur lesquelles appuient les patins.

En plus des précautions prises pour obtenir une bonne répartition des charges, nous nous sommes ef- 
forcés de réduire des déformations d'origine thermique ou mécanique. Une isolation thermique des patins diminue les gradients de température. Une circulation d'huile efficace, assurée par un groupe moto-pompe extérieur alimenté à partir des auxiliaires de la centrale, permet d'égaliser les températures autour de la couronne des patins et dans le circuit d'huile.

En ce qui concerne l'étude théorique des pivots, la méthode de calculs que nous utilisons traite le problème tridimensionnel de la lubrification des patins en secteur. Les calculs consistent à résoudre numériquement les équations de la thermo-hydrodynamique des fluides visqueux pour une loi donnée de variation de la viscosité en fonction de la température. Les déformations thermiques et mécaniques du patin et du grain mobile, déterminées à l'aide de programmes associés, sont réintroduites dans le calcul thermo-hydrodynamique du film. On itère jusqu'à la stabilisation des résultats à $1 \%$ près : en général trois calculs thermiques et deux calculs de déformation suffisent.

Le programme donne:

- la colline des pressions;

- la répartition des températures dans le métal ;

- la variation des épaisseurs de film d'huile.

- les déformations du patin.

A titre d'exemple, nous donnons en figure 7 la colline des pressions déterminée pour le pivot de Cabora Bassa sous la charge de $2100 \mathrm{t}$ correspondant aux conditions d'essais (charge maximale de calcul $2250 \mathrm{t}$ pour la puissance maximale de $485 \mathrm{MW}$ ).

Pour contrôler la validité du programme de calcul des pivots et permettre d'ajuster les différents paramètre utilisés dans ce programme (coefficients d'échange thermique, température moyenne de l'huile à l'entrée), nous avons effectué différentes mesures en 1979 :

- à Cabora Bassa (Mozambique) sur 2 pivots d'une turbine Francis de 2250 t de charge ;

- à Salignac (France) sur un pivot d'une turbine Kaplan de 800 t de charge.

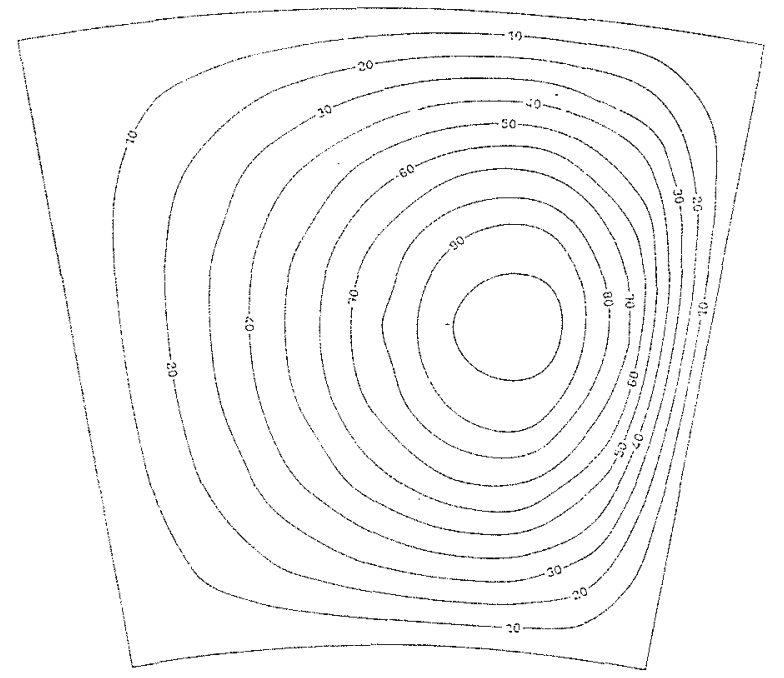

Figure 7. - Centrale de Cabora Bassa - Pivot à 14 patins Colline des pressions en bars.
Les mesures ont porté sur :

-- les épaisseurs du film d'huile ;

- les pressions sur le patin ;

-- les températures dans le métal du patin;

-.. la poussée axiale ;

- la pression dans les membranes.

Elles mettent en évidence une bonne concordance avec les calculs théoriques.

\section{Systèmes de réglage}

La régulation des turbines hydrauliques exige des connaissances multidisciplinaires allant des domaines de l'électronique à ceux de l'hydraulique. Une grande expérience nous permet d'offrir des matériels de haute qualité pour les systèmes à réglages simples ou multiples.

Dernier-né des régleurs électroniques de notre conception, le RAPID 77 réalise une régulation par action proportionnelle, intégrale et dérivée qui donne, pour la généralité des cas, une excellente qualité de réglage. Il met au service de la régulation toutes les ressources de l'électronique moderne. En effet, sa conception fait un usage systématique des circuits intégrés d'excellente fiabilité.

Les groupes de la centrale de Porto Primavera, équipés de régleurs RAPID 77, présentent des avantages particuliers suivants :

- suppression de l'alternateur pilote ;

- asservissements électriques, éliminant jeux et frottements des transmissions mécaniques;

- systèmes copiants électriques incorporés à l'armoire du régleur pour la commande des pales Kaplan ;

- paramètres de réglage ajustables pendant la marche du groupe.

Le système de régulation comprend d'autre part :

- un transducteur électrohydraulique, ou actionneur, qui transforme le signal électrique en variation de débit et constitue le premier étage de puissance ;

- l'ensemble de la servo-commande hydraulique du distributeur qui réalise un étage d'amplification hydraulique important et fournit la puissance nécessaire à la mancuvre des servomoteurs (débit maximal pour Porto Primavera $=301 / \mathrm{s})$;

- des variomètres qui sont des transducteurs déplacement - tension.

Les circuits vannage et pales ont chacun leur chaîne de régulation ; la position des pales est copiée sur celle du vannage.

La conjugaison pales-vannage, en fonction de deux variables : ouverture du distributeur et chute, s'effectue au moyen d'une came électrique. La fonction des deux variables à réaliser est schématisée par une famille de courbes à 2 paramètres dans le plan.

Les grandeurs d'entrée, ouverture du distributeur et hauteur de chute, existent sous la forme de tensions proportionnelles, variables entre 0 et $10 \mathrm{~V}$. On peut réaliser un ensemble de circuits électroniques qui permettent d'obtenir un signal électrique respectant la loi de conjugaison déterminée, à partir des grandeurs d'entrée. Si on se fixe la tension proportionnelle à la chute, les circuits électroniques choisis permettent 
l'approximation d'une courbe quelconque par une série de segments de droites ( 5 au maximum). Par extension, pour deux variables d'entrée indépendantes, on peut réaliser l'approximation d'une surface quelconque par une série de polygones.

L'énergie nécessaire à la commande des pales et du vannage est fournie par de l'huile à la pression maximale de 45 bars, emmagasinée dans un réservoir commun aux circuits pales et vannage, mais réalisé en deux parties : un réservoir d'air comprimé et un réservoir air-huile interconnectés. Le dimensionnement de ces réservoirs permet, sans que les pompes de régulation entrent en service, les fonctionnements successifs suivants :

- une fermeture complète du vannage et la fermeture correspondante des pales;

- une ouverture totale du vannage et l'ouverture correspondante des pales;

- la fermeture complète du vannage et des pales.

Chaque groupe dispose d'un compresseur et d'un réservoir d'air ; toutefois, une intercommunication existe entre les groupes.

Le choix des temps de manœuvre des pales et du vannage dépend des valeurs maximales des surpressions admises et de la limitation de la dépression sous la roue.

Pour estimer l'évolution des pressions et la variation de vitesse du groupe au cours des fonctionnements transitoires, un programme de calculs sur ordinateur prend en compte tous les paramètres des adductions amont et aval du groupe, ainsi que les caractéristiques de la colline en déconjugaison et celles du régleur

Les temps de manœuvre calculés sont optimisés au cours de la mise en route.

A titre d'exemple, à Porto Primavera, les temps de manœuvre sont de l'ordre de

- 10 s pour la fermeture du distributeur;

- 20 s pour l'ouverture du distributeur ;

- $70 \mathrm{~s}$ pour l'ouverture et la fermeture des pales.

\section{Conclusions}

L'analyse rapide, faite à propos des éléments principaux des turbines Kaplan de grandes dimensions, l'exposé d'ensemble des recherches sur les écoulements et des méthodes de calculs montrent que nous disposons des moyens nécessaires à la conception et au dimensionnement des turbines Kaplan de fortes puissances. Nous pouvons envisager avec succès l'étude et la conception de roues dont le diamètre dépasse $10 \mathrm{~m}$ et de pivots dont la charge peut aller au-delà de 4000 tonnes.

\section{TURBINES AXIALES DU TYPE BULBE AMONT}

Il y a plus de 25 ans, E.D.F. lançait un concours d'idées sur la réduction des coûts des installations de basses chutes. A cette époque, les constructeurs réalisaient des études sur les centrales marémotrices et recherchaient de nouveaux types de machines, capables de fonctionner en turbine et en pompe, dans les deux sens d'écoulement.

Le résultat de ces recherches sur l'économie des basses chutes et des sites marémoteurs fut la mise au point des groupes axiaux du type bulbe amont. Le choix de ce genre de machine se justifiait par d'appréciables avantages. Les formes simples des conduits hydrauliques pratiquement axisymétriques permettaient d'augmenter les puissances spécifiques et apportaient à la fois une réduction des coûts des ouvrages de génie civil et du matériel électromécanique.

L'équipement du site marémoteur de la Rance et l'aménagement des grands fleuves français avec des groupes bulbes constituèrent les premiers succès dans la recherche de l'amélioration de la rentabilité des basses chutes. Quelques difficultés marquèrent la mise au point de la technique des alternateurs, difficultés pour lesquelles les techniciens trouvèrent des solutions définitives.

Actuellement, un grand nombre de centrales équipées de groupes bulbes assurent un service donnant entière satisfaction aux clients. L'installation de Rock Island sur la Columbia River, équipée de 8 unités développant chacune $54 \mathrm{MW}$ sous $12,1 \mathrm{~m}$ de chute, fonctionne depuis près de quatre ans et détient toujours le record de puissance et de dimension. On peut affirmer aujourd'hui que les limites techniques des groupes bulbes sont pratiquement identiques à celles des turbines Kaplan conventionnelles.

Maitres d'œuvre et constructeurs cherchent toujours à bénéficier, sans courir de risque déraisonnable, de tout progrès des techniques utilisées au stade du projet et de la construction des installations hydroélectriques de basses chutes.

Les coûts des ouvrages de génie civil, les équipements électromécaniques, les sytèmes de commande et de contrôle restent parmi les postes principaux du passif dont l'importance relative varie évidemment suivant les types d'aménagements. Au passif de l'opération figurent aussi les intêrêts intercalaires qui dépendent des délais de construction, de montage et de mise en exploitation.

Une étude d'ensemble de tous ces aspects devrait conduire à de nouvelles améliorations sensibles de la rentabilité globale de l'opération. La recherche de l'optimum économique varie à l'infini suivant les cas envisagés depuis l'équipemont des grands fleuves et des sites marémoteurs jusqu'aux petits aménagements ; les premiers exigent la collaboration de toutes les disciplines techniques concernées, les seconds peuvent rester à l'échelle d'un maître d'œuvre ou d'un constructeur.

Des progrès appréciables devraient résulter d'une meilleure collaboration entre maitres d'œuvre et constructeurs. Les premières analyses faites à l'occasion de projets d'installations marémotrices laissent espérer des gains très sensibles sur les coûts et les délais. Ces études font ressortir que l'économie maximale exige une remise en cause des technologies de construction des ouvrages et du matériel électromécanique. Il va sans dire, que pour toutes les dispositions envisagées, les qualités des équipements hydro-électriques, notamment la souplesse d'exploitation, la grande disponibilité, l'entretien réduit, etc., doivent être conservées. 
Installations équipées de groupes bulbes de grandes puissances

Nous nous proposons dans la présente étude de faire le point de la technique des groupes bulbes et d'analyser, en bénéficiant des connaissances acquises, les nouvelles possibilités que peut apporter l'utilisation de ces machines pour l'équipement des basses chutes. Cet examen concernera la technologie, la fabrication, l'intégration du matériel électromécanique à la centrale ; il s'étendra aussi à la conception de la centrale elle-même, au montage et aux délais de mise en exploitation. 11 va sans dire que nous ne pourrons qu'esquisser les progrès envisageables et inciter tous les spécialistes à la réflexion.

Nous traiterons d'abord des installations de 10 à $25 \mathrm{~m}$ de chute, caractérisées par des débits d'équipement élevés, justifiant l'utilisation de groupes axiaux de puissance unitaire importante, pouvant atteindre $70 \mathrm{MW}$ et même $100 \mathrm{MW}$.

Nous analyserons ensuite les aspects spécifiques des installations de chute inférieure à $10 \mathrm{~m}$, elles aussi caractérisées par des débits élevés. Nous examinerons les possibilités d'emploi de groupes simplifiés, munis d'alternateur entrainé par l'intermédiaire d'un multiplicateur. Nous verrons que certaines recherches d'économie ne réussiront que si l'on remet en cause les technologies des ouvrages de génie civil et du matériel électromécanique.

Nous donnerons enfin quelques informations sur l'équipement des installations marémotrices actuellement en cours d'étude.

\section{Installations de 10 à $25 \mathrm{~m}$ de chute à débits d'équipe- ment élevés}

De nombreuses centrales équipées de groupes bulbes assurent aujourd hui un service donnant entière satisfaction. Les limites techniques de ces machines, comparables à celles des Kaplan verticales conventionnelles dépendent uniquement de l'économie d'ensemble des projets.

Le tableau I donne les principales caractéristiques des groupes bulbes de grandes puissances unitaires en exploitation depuis plusieurs années ou actuellement en cours de montage.

Les deux premières installations sont en exploitation, la première depuis près de 4 ans, la seconde depuis plus d'un an.
Les groupes équipant ces centrales, disposés en conduits, comportent tous un double réglage, mais diffèrent par les conceptions des structures porteuses et des lignes d'arbre.

\section{Evolution des structures support}

A Rock Island (Fig. 8) les groupes reposent :

- sur deux piles profilées en béton, précontraintes verticalement et placées au droit du stator de l'alternateur. La carcasse du stator, munie de pattes, se fixe par boulonnage sur ces piles.

- sur un avant-distributeur à 6 avant-directrices creuses fortement ancrées dans le béton.

Piles et avant-distributeur reprennent les efforts axiaux, radiaux et les couples.

Cette structure hyperstatique de grande rigidité présente l'inconvénient d'être sensible aux variations de température. Les dilatations ou contractions relatives de la caracasse de l'alternateur créent des efforts axiaux et radiaux qu'il faut prendre en compte.

Pour éviter ces forces parasites, nous adoptons des structures d'une rigidité d'ensemble satisfaisante sur lesquelles les écarts de température n'ont pratiquement aucune influence. Les installations de Chautagne, Belley et Cakovec bénéficient de ces nouveaux types de structures.

A Chautagne et Belley (Fig. 9) la structure support se caractérise par :

- en amont de l'alternateur, une ogive munie de deux bras verticaux profilés et de section importante (largeur : $1,5 \mathrm{~m}$; longueur : $5.6 \mathrm{~m}$ );

- en aval de l'alternateur, un anneau tronconique très rigide et 6 buttons radiaux précontraints.

L'ogive amont et ses bras reprennent les couples, les efforts axiaux et radiaux. Les buttons précontraints limitent fortement les déplacements radiaux, augmentent les fréquences propres, mais ne s'opposent pas aux déformations axiales du stator.

A Cakovec, comme à Crestuma (Fig. 10) l'avantdistributeur muni de 6 avant-directrices dont deux creuses disposées dans le plan vertical, constitue la structure support principale et reprend les couples, les efforts axiaux et pratiquement la totalité des efforts radiaux. En amont, au droit de logive, 3 buttons (1 vertical et 2 horizontaux) assurent des appuis radiaux secondaires qui améliorent le comportement vibratoire des groupes.

\begin{tabular}{|l|c|c|c|c|c|}
\hline \multicolumn{7}{|c|}{ Tableau I } \\
\hline \multicolumn{1}{|c|}{ Installations } & $\begin{array}{c}\text { Nbre de } \\
\text { groupes }\end{array}$ & N en MW & h en $\mathrm{m}$ & $\begin{array}{c}\mathrm{n} \text { en } \\
\text { tr/min }\end{array}$ & D roue en mm \\
\hline Rock Island (USA) & 8 & 54 & 12,1 & 85,7 & 7400 \\
Chautagne (FR) & 2 & 46,6 & $14,67-16,15$ & 107 & 6400 \\
Belley (FR) & 2 & 46,7 & $14,7-17,02$ & 107 & 6400 \\
Cakovec (YUG) & 2 & 42,2 & 18,5 & 125 & 5400 \\
Crestuma (Portugal) & 3 & 40,2 & 11,25 & 83,3 & 6800 \\
\hline
\end{tabular}




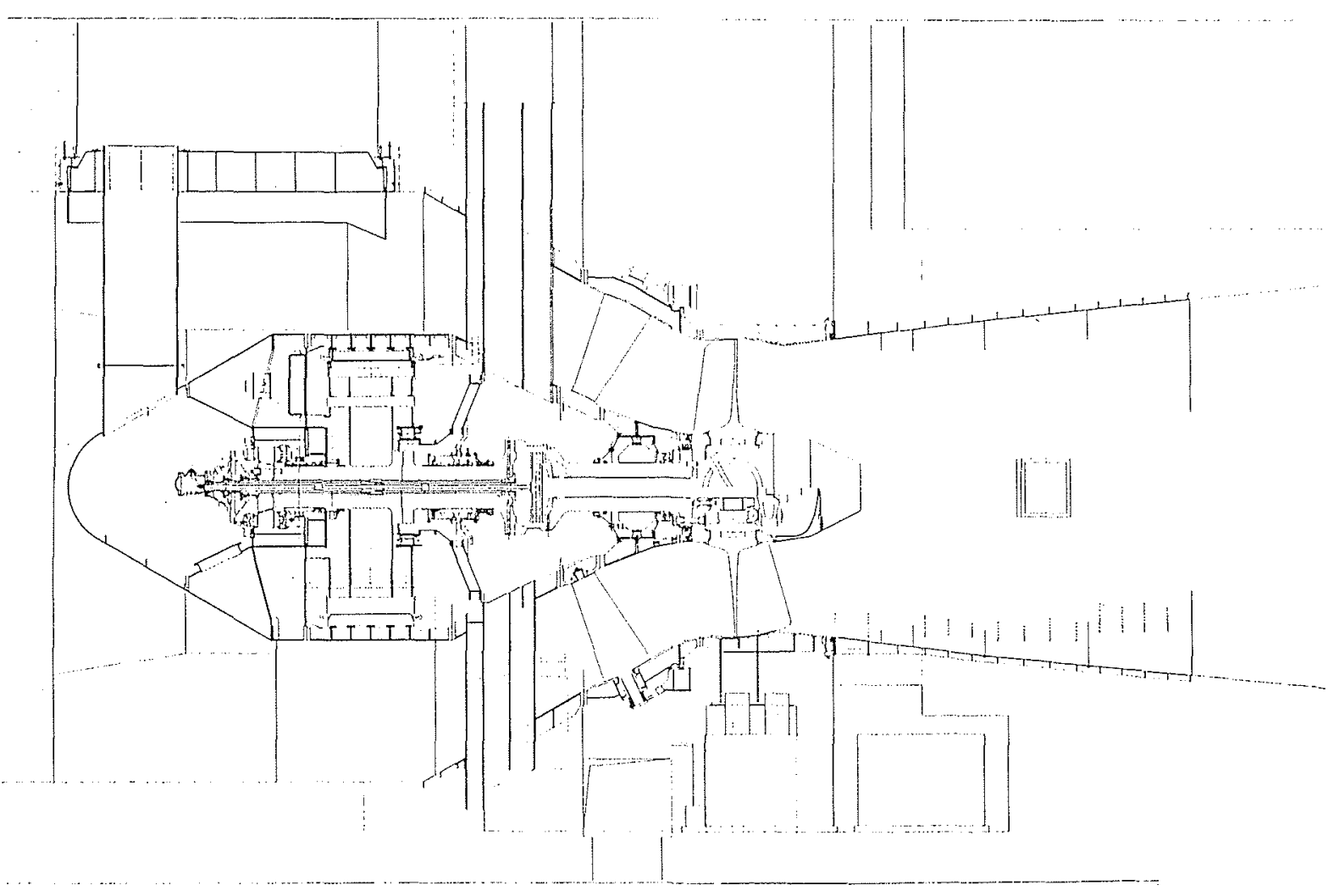

Figure 8. - Centrale de Rock Island - Coupe de la turbine bulbe de $54 \mathrm{MW}$.

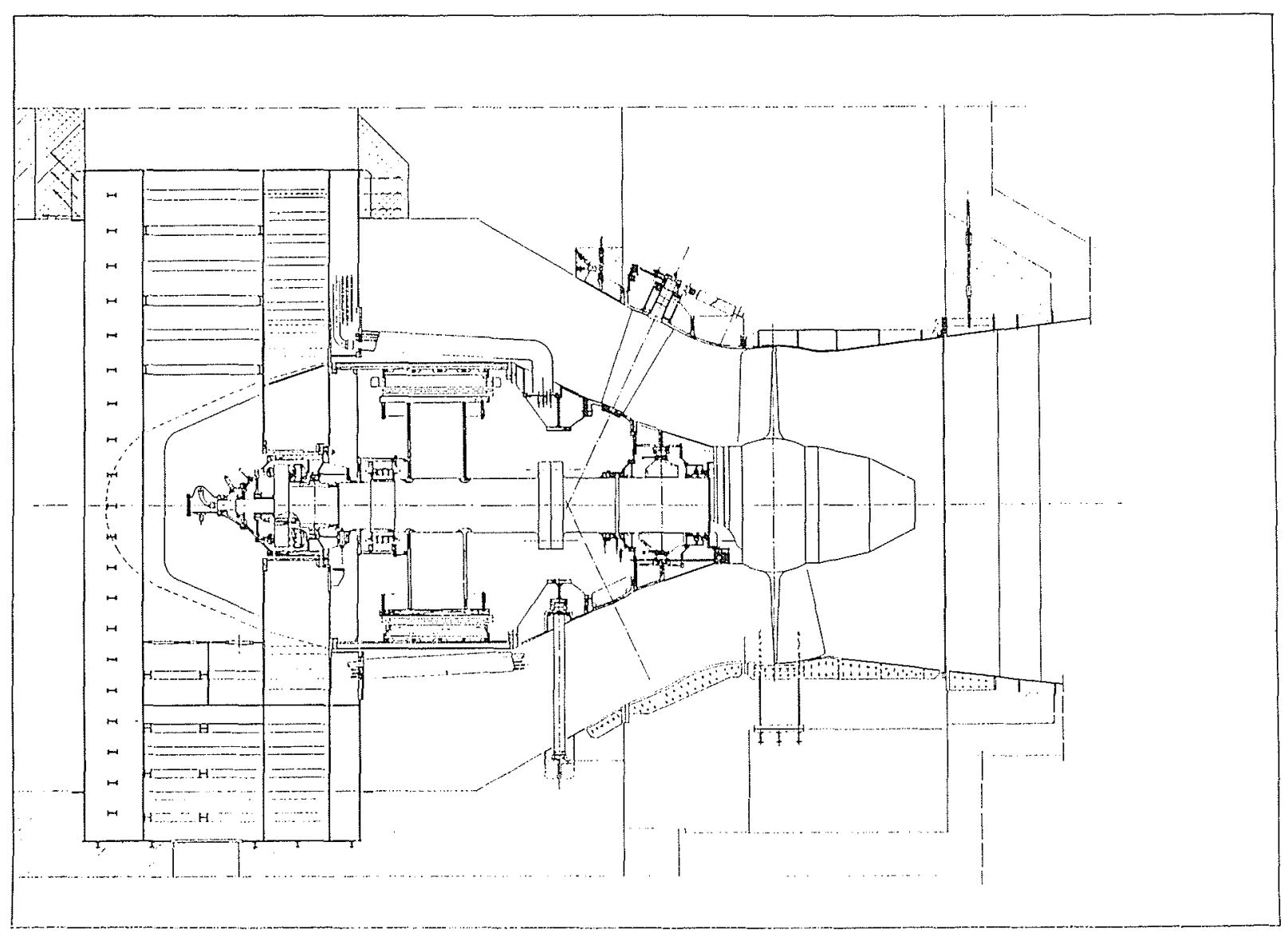

Figure 9. - Centrales de Chautagne et Belley - Coupe de la turbine bulbe de $46,7 \mathrm{MW}$. 


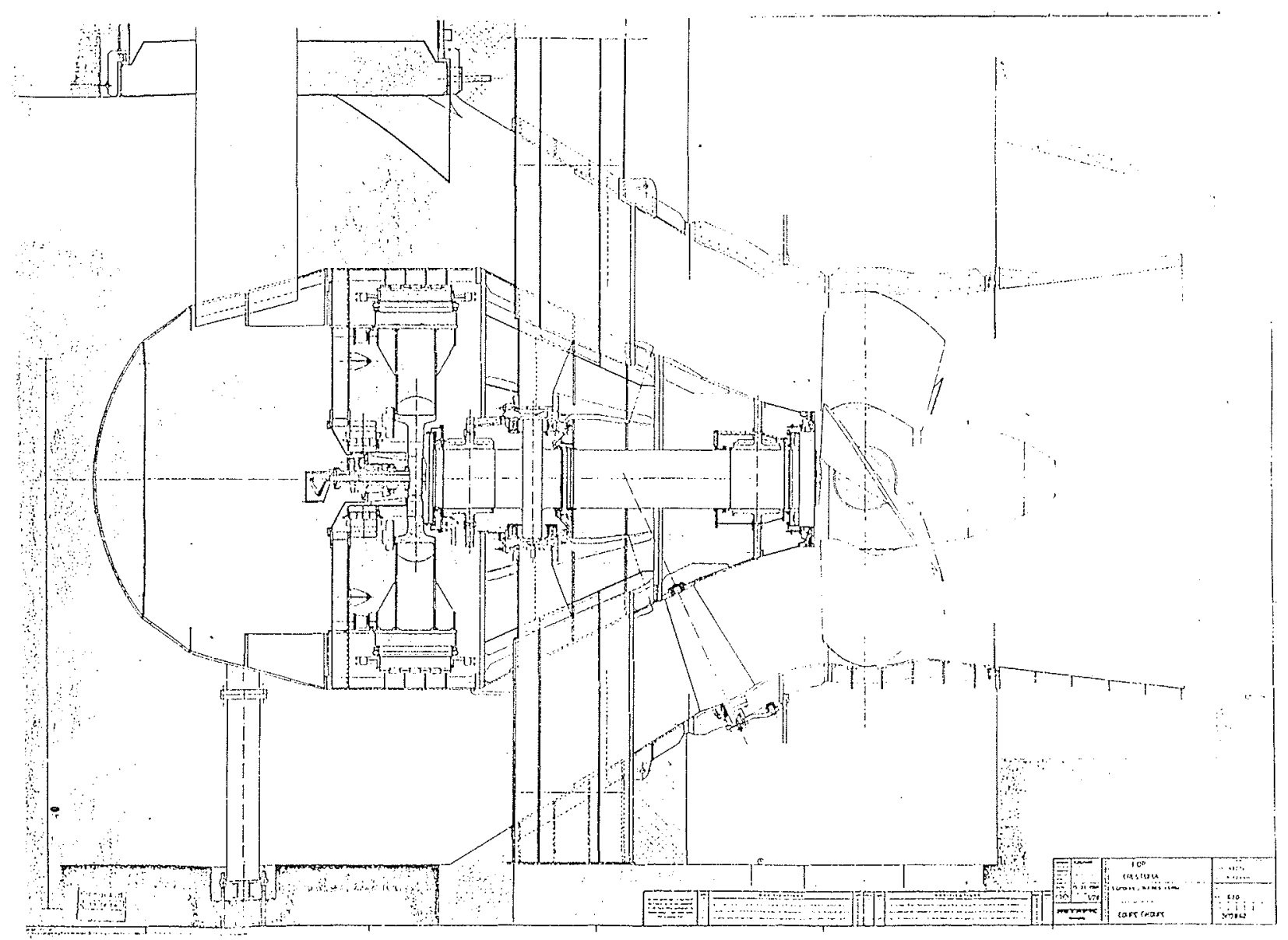

Figure 10 - Centrale de Crestuma - Coupe de la turbine bulbe de 40,2 MW.

\section{Lignes d'arbres}

L'étude de la ligne d'arbre de Rock Island, guidée par le souci de ne pas charger bien au-delà de 200 tonnes le palier turbine, nous a amenés à choisir une solution à trois paliers porteurs. Rappelons que la masse totale des parties tournantes d'un groupe atteint 365 tonnes. Le palier turbine à patins supporte 200 tonnes environ (Fig. 11), les deux autres moins chargés restent du type cylindrique à coussinet.

A Chautagne, Belley et Cakovec, comme dans la plupart de nos réalisations en bulbes, la ligne d'arbre repose sur deux paliers disposés respectivement à l'amont de la turbine et de l'alternateur. La butée et la contrebutée situées au droit du support principal transmettent directement les poussées aux ancrages dans le béton.

La coupe des groupes de Crestuma (Fig. 10) montre une conception qui comporte un alternateur monté en porte-à-faux à l'extrémité amont d'un arbre unique reposant sur deux paliers.

Cette disposition s'applique à des machines pour lesquelles le rapport entre la longueur de fer et le diamètre d'alésage du rotor reste limité.

Le choix de l'arbre unique exige qu'on place le servomoteur des pales dans le moyeu de la roue à l'aval du plan des pales. L'adoption de pressions de régulation élevées facilite l'étude de cette conception mécanique.

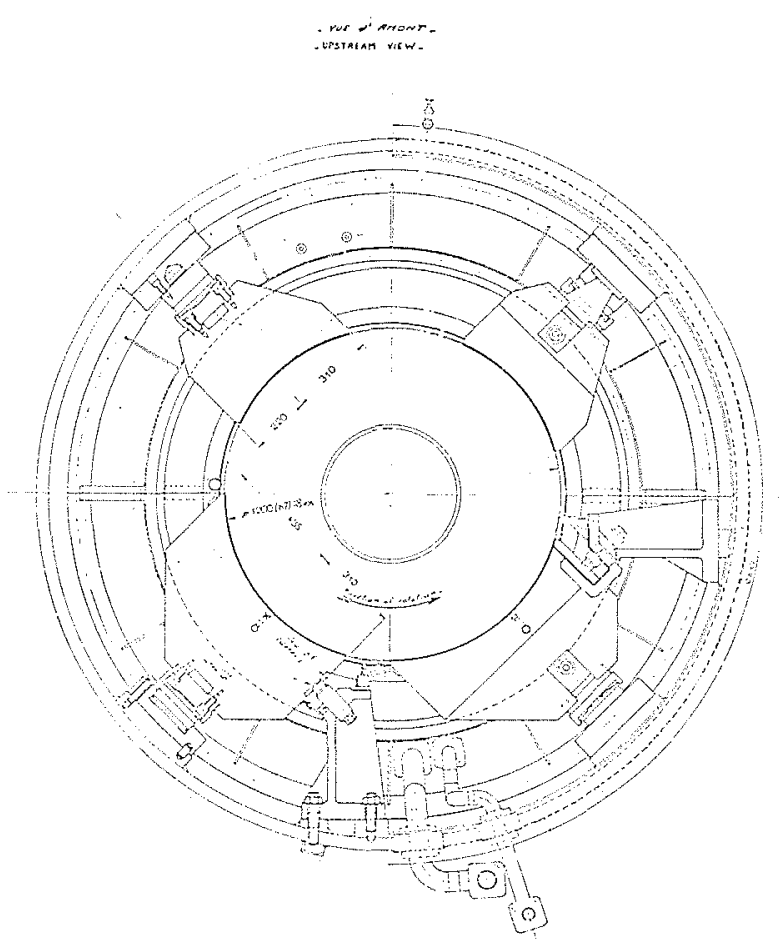

Figure 11. - Centrale de Rock Island - Coupe du palier turbine. 
L'arrangement avec arbre unique et rotor alternateur en porte-à-faux présente l'avantage de répartir au mieux les charges entre les deux paliers. Ainsi, pour les groupes de Crestuma, on a pu adopter deux paliers identiques supportant environ 120 tonnes, tandis que les machines de Chautagne comportent un palier à patins de près de 200 tonnes et un coussinet cylindrique de 65 tonnes.

La conception avec arbre unique et alternateur en porte-à-faux présente en plus des avantages très réels pour le montage :

- un outillage approprié permet d'introduire et de mettre en place, dans l'avant-distributeur et le cône turbine, l'ensemble de l'arbre, des paliers et des butées. - l'ensemble de l'arbre étant en place, le montage des autres organes de la turbine (distributeur, roue, ceinture...) s'effectue au droit de la fosse aval du groupe pendant que, simultanément se fait au droit de la fosse amont, le montage du rotor, du stator de l'alternateur et de l'ogive.

\section{Installations de 4 à $10 \mathrm{~m}$ de chute à débits d'équipe- ment élevés}

La rentabilité des aménagements, caractérisés par des chutes comprises entre 4 et $10 \mathrm{~m}$ et des débits d'équipement importants, nécessite le développement de conceptions d'ensemble économiques conduisant à une réduction des coûts de génie civil et du matériel électromécanique. Des đispositions commencent à se préciser à la suite d'études effectuées pour des centrales au fil de l'eau ou des installations marémotrices. Nous traiterons des solutions nouvelles suivantes :

- emploi de groupes à système de réglage simplifié ; - utilisation de multiplicateurs de vitesse.

L'équipement électromécanique représente, pour les basses chutes, une part importante de l'investissement total ; l'adoption de machines simplifiées de performances satisfaisantes peut apporter une réduction sensible du coût. Le prix de la turbine varie sensiblement suivant le mécanisme de vannage choisi pour le contrôle du débit.

Le réglage de la charge peut se faire par une manœuvre combinée des aubes du distributeur et des pales de la roue. Nous désignerons ces turbines en abrégé par DM-PM.

Le contrôle de la puissance, pour une roue à pales fixes, peut s'effectuer par la commande de la rotation des directrices. Nous adopterons l'abréviation DM.PF pour ces machines.

Avec un distributeur à aubes fixes, les variations de charge s'opèrent par manœuvre de l'angle d'inclinaison des pales de la roue. La dénomination DF-PM se rapportera à ces turbines.

Signalons que les centrales caractérisées par des chutes pratiquement constantes peuvent être équipées de machines ne comportant aucun organe de vannage. Elles fournissent une puissance constante à la vitesse de synchronisme pour une chute donnée. La mise en route et l'arrêt de ces turbines s'effectuent à l'aide d'une vanne située, en général, à l'aval de la roue.
Les collines caractéristiques 12 (1), 12 (2), 12 (3), 12 (4) relatives à des groupes du type bulbe amont se rapportent respectivement à des turbines DM-PM, DF-PM et DM-PF, les deux dernières correspondent à des angles d'inclinaison de la roue de $25^{\circ}$ et $30^{\circ}$.

Les machines DM-PM donnent les meilleures performances hydrauliques d'ensemble : puissances et vitesses spécifiques, rendements, moindre sensibilité à la cavitation, etc. Les collines des turbines DF-PM, bien que moins favorables que celles des DM-PM, ont des rendements aux charges partielles encore acceptables, tandis que pour les groupes DM-PF, les rendements chutent brutalement lorsque la charge baisse. Rappelons que le débit de marche à vide des turbines DM-PF atteint 30 à $40 \%$ du débit nominal. Si l'on désire régler la charge de la marche à vide à la pleine puissance il est donc préférable d'adopter des machines du type DM-PM ou DF-PM, d'autant plus que le comportement de ces turbines reste très calme quelle que soit la charge, ce qui n'est pas le cas des machines DM-PF.

L'adoption de groupes à systèmes de réglage simplifiés, dont nous donnerons un exemple de réalisation concernant l'installation de W.T. Love, constitue une des voies tendant à diminuer le prix du matériel électromécanique. Nous verrons que ces réductions de coût concernent̂ le génie civil et les machines.

Les unités destinées aux aménagements envisagés peuvent comporter des turbines dont les diamètres des roues varient de 6 à $8 \mathrm{~m}$ pour des vitesses de rotation allant de 40 à $50 \mathrm{tr} / \mathrm{min}$. La conception des alternateurs à attaque directe devient alors très délicate. Leur dimensionnement risque de conduire à une augmentation sensible du diamètre maximal du distributeur de la turbine et entraîner une majoration importante du prix de cet organe essentiel, ainsi qu'à un allongement de la machine et à un accroissement de l'entr'axe des groupes. Les progrès réalisés dans la conception et la fabrication des multiplicateurs de vitesse et l'amélioration considérable de leur fiabilité permettent d'envisager leur utilisation pour l'entraînement d'alternateurs rapides de technique plus conventionnelle et éprouvée. Nous décrirons un exemple d'application et préciserons les avantages apportés par cette disposition.

Installation de W.T. Love : groupes à distributeur fixe et pales mobiles

Les trois groupes équipant cette centrale, disposés en chambre d'eau, comportent un distributeur fixe et une roue à pales orientables (Fig. 13).

Les parties tournantes reposent sur deux paliers, situés entre le rotor de l'alternateur et la roue de la turbine qui se trouvent tous les deux en porte-à-faux.

Les principales caractéristiques de ces machines sont :

- Puissance nominale : $24,5 \mathrm{MW}$

- Vitesse : $90 \mathrm{tr} / \mathrm{min}$

- Hauteur de chute nominale : $8,4 \mathrm{~m}$

- Débit nominal $: 315 \mathrm{~m}^{3} / \mathrm{sec}$

- Diamètre de roue $\quad: 6100 \mathrm{~mm}$.

Les chutes nettes exploitables varient de 1,2 à $9,15 \mathrm{~m}$; sous cette dernière, la puissance maximale atteint $26,7 \mathrm{MW}$. 


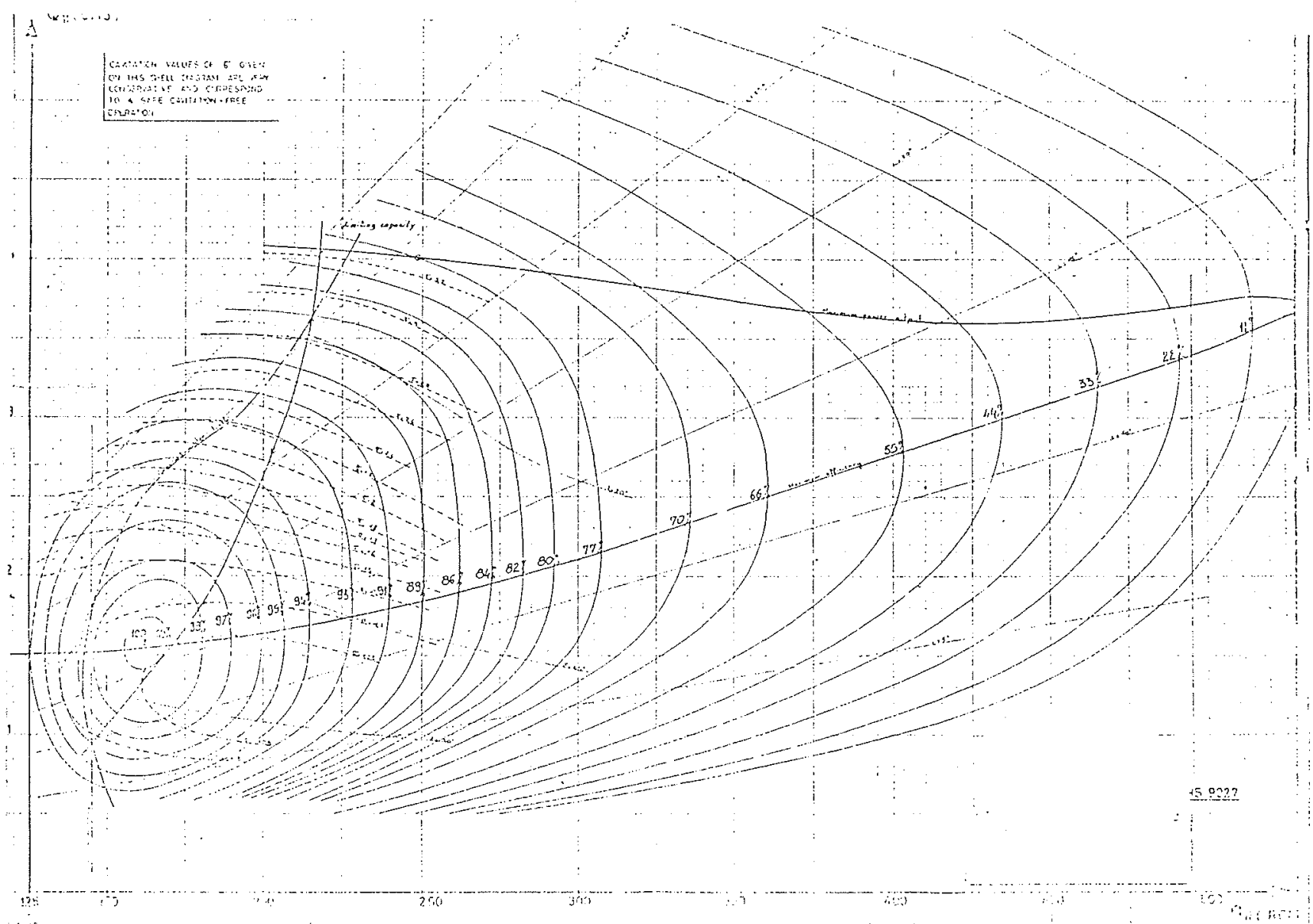

Figure 12 (1). - Collines caractéristiques hydrauliques d'une machine DM-PM.

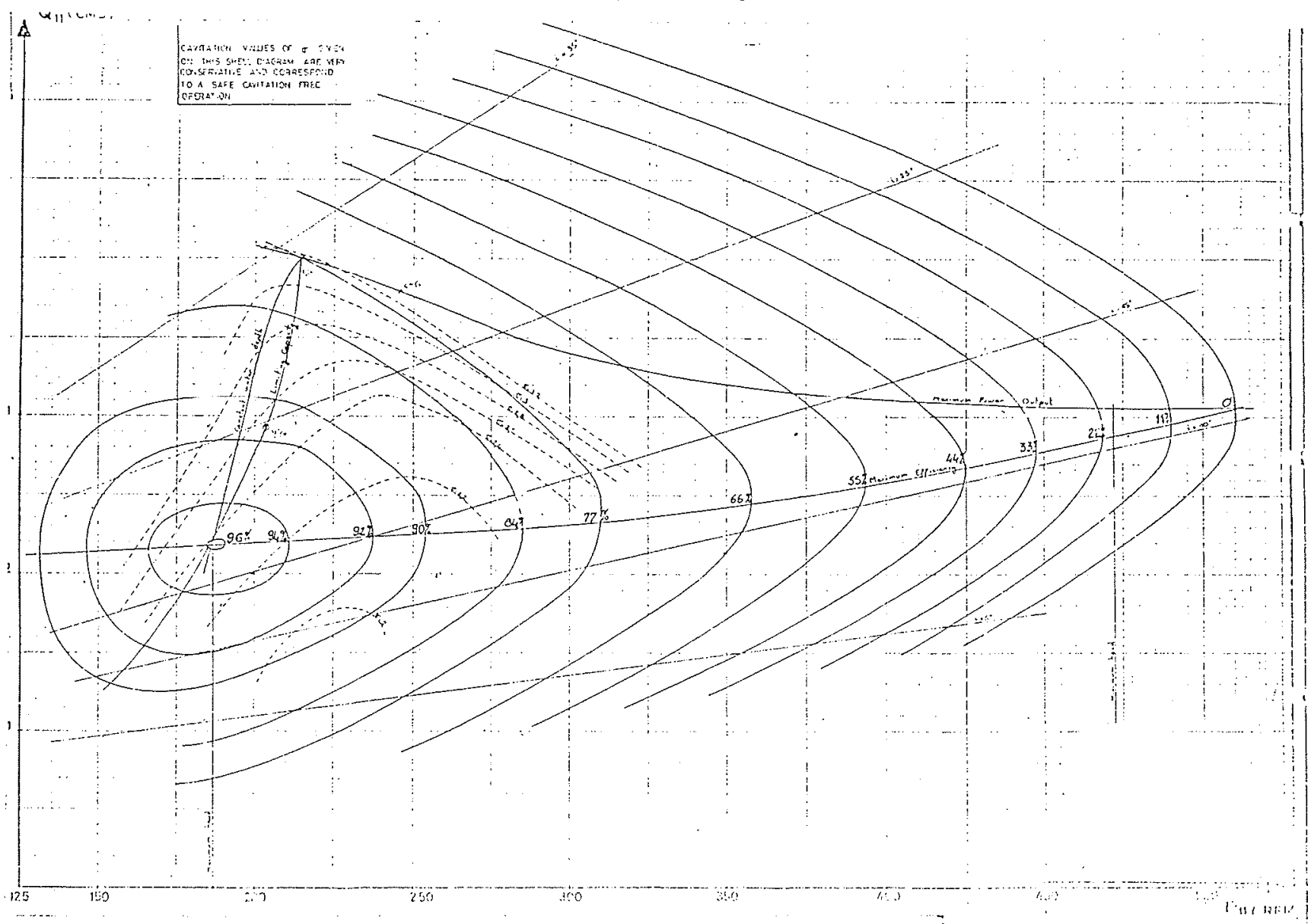

Figure 12 (2). - Collines caractéristiques hydrauliques d'une machine DF-PM. 


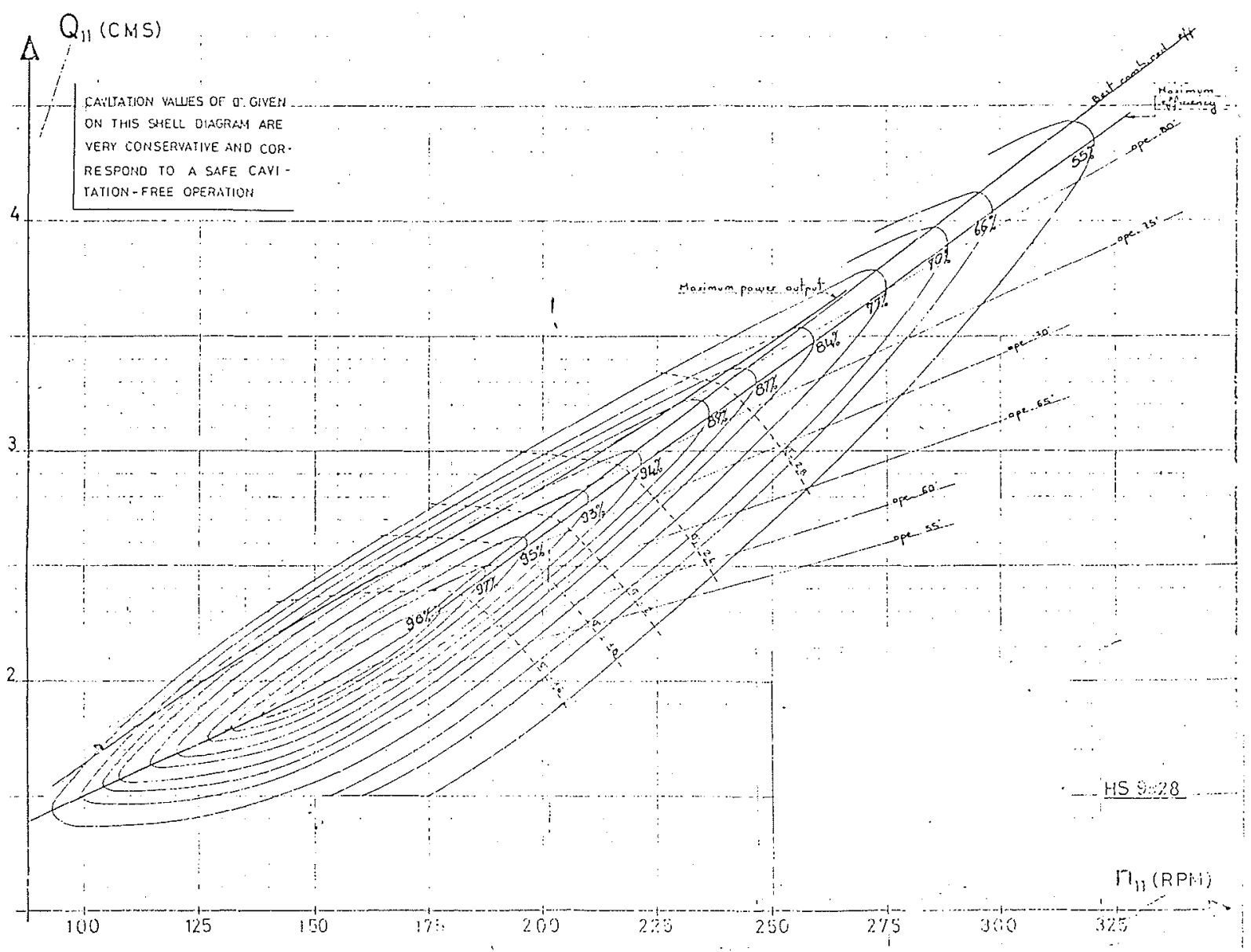

Figure 12 (3). - Collines caractéristiques hydrauliques d'une machine DM-PF $-\hat{\mathbf{i}}=25^{\circ}$.

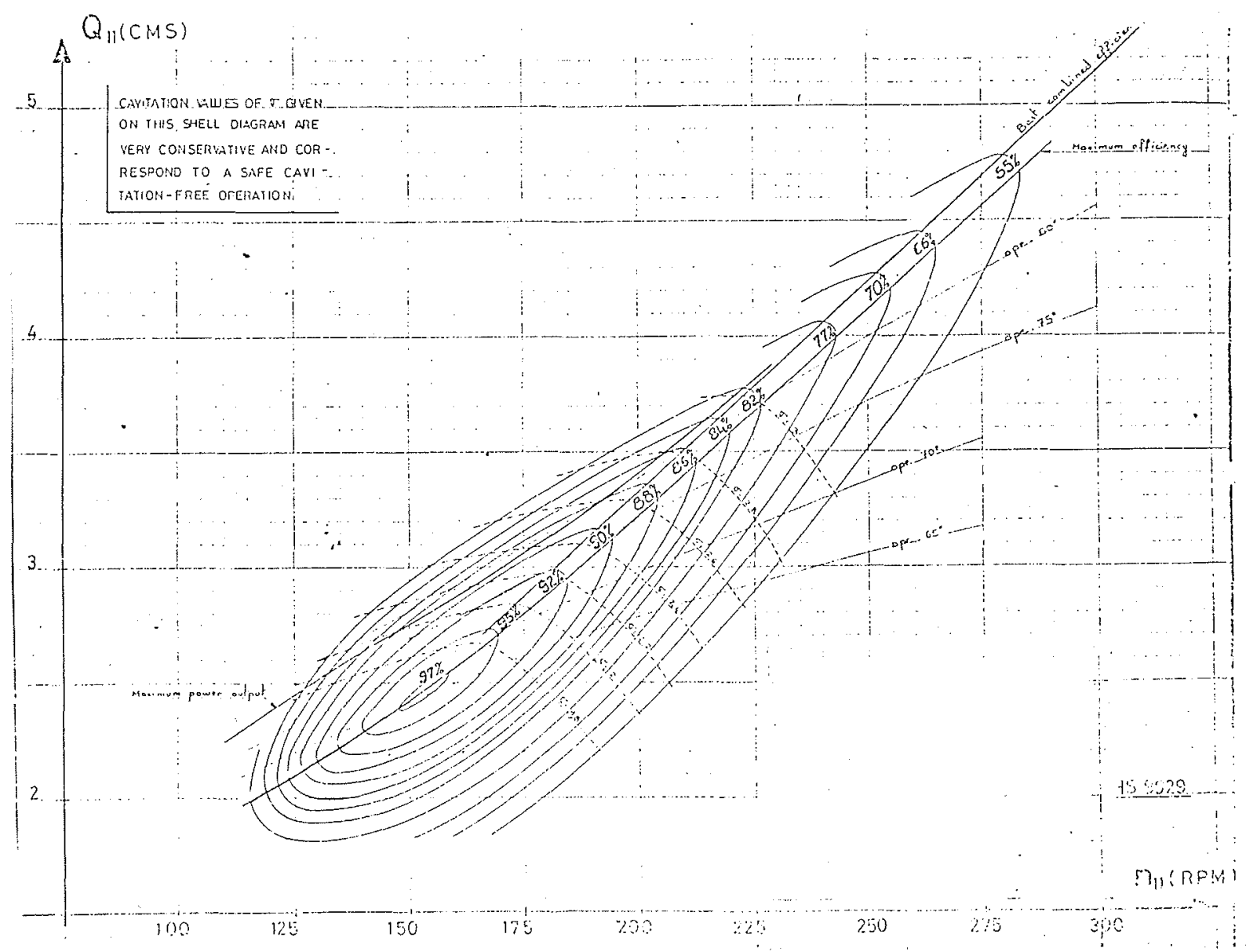

Figure 12 (4). - Collines caractéristiques hydrauliques d'une machine DM-PF $-\hat{1}=30^{\circ}$. 


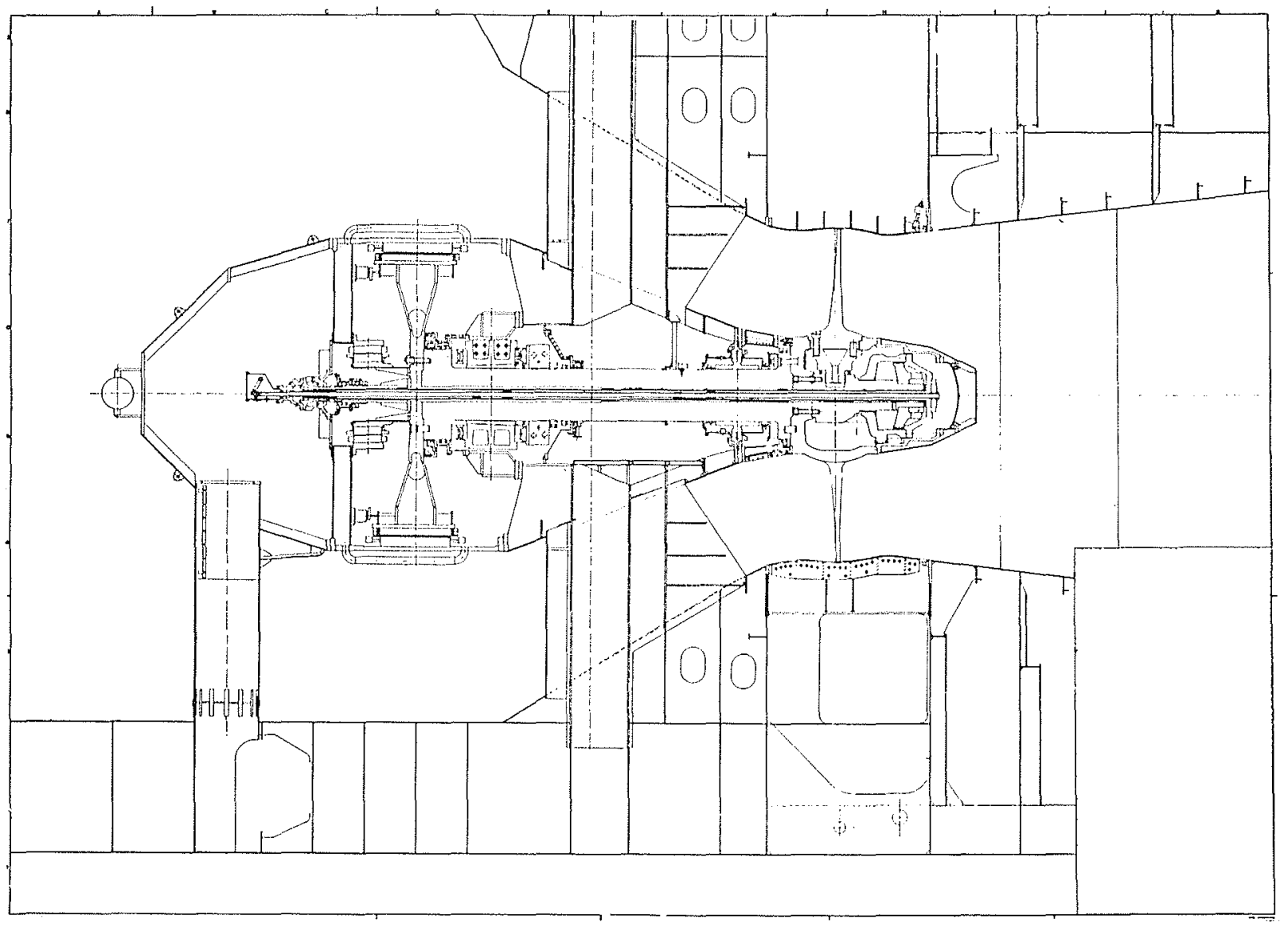

Figure 13. - Centrale W.T. Love - Coupe de la turbine bulbe de 24,3 MW.

Les alternateurs pressurisés à 1 bar sont dimensionnés pour une puissance nominale de 23,9 MVA, sous $4160 \mathrm{~V}$ à $60 \mathrm{~Hz}$, avec un diamètre extérieur de carcasse du stator de $5725 \mathrm{~mm}$.

Nous renvoyons le lecteur aux publications qui décrivent la Centrale de W.T. Love. L'usine préfabriquée et complètement équipée dans les chantiers navals d'Alsthom Atlantique à Saint-Nazaire a été amenée sur le site après la traversée de l'Atlantique et la remontée du Mississipi et de l'Ohio.

Les structures support du groupe comprennent un appui principal de grande rigidité : l'avant-distributeur et des buttons situés au droit de l'ogive amont. Les déplacements par rapport à l'appui principal, déjà très faibles, se trouvent encore réduits par l'action des buttons qui augmentent ainsi les fréquences propres des modes de vibrations possibles autour de l'avantdistributeur.

On voit sur la figure 13 que ce type de support permet, d'une part, de simplifier très sensiblement les structures du groupe et leur ancrage et d'autre part, de réduire le puits de montage et démontage au droit de la roue. Enfin, remarquons qu'aucun mécanisme de vannage ne se trouve à l'extérieur du bulbe ; toutes les parties mécaniques délicates se situent à l'intérieur.

Nous ne décrirons pas les dispositions mécaniques que montre clairement la figure 13. Signalons seulement, que pour cet arrangement, tous les organes principaux du groupe restent accessibles. Le bras inférieur à l'amont donne accès aux alvéoles de distribution d'huile sous pression, au servo-moteur des pales, à l'excitatrice, au système de freinage et permet certains démontages des enroulements du stator de l'alternateur. Les avant-directrices creuses permettent d'accéder aux paliers et à la butée. Le joint tournant d'arbre est accessible de l'intérieur du conduit hydraulique.

Enfin, la disposition du servo-moteur dans l'ogive de la roue rend possible le démontage des tubes d'admission d'huile sans toucher à la ligne d'arbre.

La vanne aval qui constitue l'organe de sécurité principal est directement contrôlée par le système de régulation; elle assure les fonctions principales suivantes :

- participation au couplage sur le réseau ; de très nombreuses réalisations en ont montré le bon comportement - protection contre les survitesses élevées, optimisation des lois de manceuvre pour limiter les transitoires - marche en déchargeur

La descente de la vanne s'effectue par gravité.

Le système de régulation, équipé d'un régleur RAPID, exécute automatiquement toutes les fonctions de démarrage, d'arrêt, de marche en déchargeur et assure le réglage de la fréquence.

\section{Groupes munis d'un multiplicateur de vitesse}

La figure 14 se rapporte à un projet de centrale aux U.S.A. qui comprendrait 10 turbines d'un diamètre de 


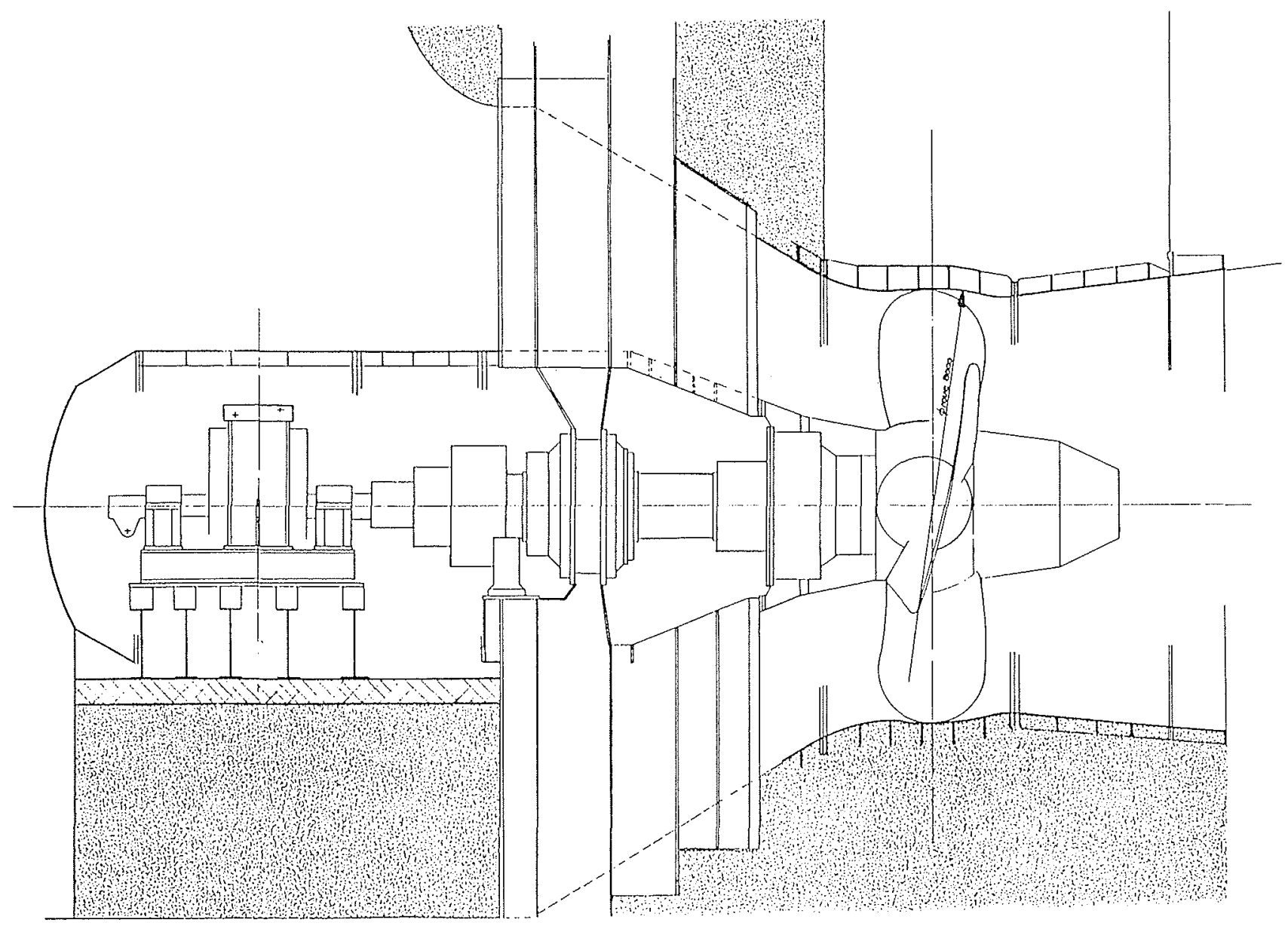

Figure 14. - Projet de centrale aux U.S.A. - Coupe d'une turbine de 11,5 MW.

$8 \mathrm{~m}$ développant $11,5 \mathrm{MW}$ sous $3,60 \mathrm{~m}$ de chute en tournant à $48 \mathrm{tr} / \mathrm{min}$.

La turbine entraîne, par l'intermédiaire d'un multiplicateur planétaire à 2 étages, un alternateur de $12,5 \mathrm{MVA}$ tournant à $450 \mathrm{tr} / \mathrm{min}$. L'ensemble multiplicateur-alternateur, installé dans un bulbe cylindrique de $5,8 \mathrm{~m}$ de diamètre, se fixe sur le cône intérieur du distributeur, d'une part et sur une pile inférieure, d'autre part. Les appuis principaux de la machine, constitués par deux avant-directrices creuses, situées dans le plan vertical et la pile placée sous le bulbe, transmettent les charges et couples aux ouvrages de génie civil. La ligne d'arbre se subdivise en deux tronçons liés par le multiplicateur de vitesse. L'arbre-turbine repose sur deux paliers et comporte une butée axiale à double effet supportant la poussée hydraulique qui ne se transmet pas au multiplicateur. Celui-ci, accouplé rigidement sur l'arbre-turbine comporte des parties statoriques liées aux structures fixes de la machine par un dispositif autorisant leurs déplacements et rotations suivant 5 degrés de liberté. Les mouvements de l'arbre-turbine, lors des régimes transitoires ou à l'emballement, compensés par ce dispositif, n'entraînent aucune surcharge des dentures qui ne transmettent que le couple moteur. Un accouplement à dents assure l'entraînement de l'alternateur, de sorte que les arbres de la turbine et de l'alternateur sont découplés tant pour la flexion que pour les déplacements axiaux.
Un puits ménagé dans l'avant-directrice creuse supérieure donne accès à l'intérieur du bulbe qui comporte un plancher de circulation permettant une visite complète de la machine, même en rotation. On utilise l'avant-directrice inférieure comme réservoir d'huile et passage des tuyauteries diverses.

Le tronçon cylindrique du bulbe est coupé en deux parties par un plan diamétral horizontal. Après démontage de l'ogive amont et de la demi-coque supérieure $\mathrm{du}$ bulbe, on atteint directement l'atlemateur et/ou le multiplicateur qu'on démonte facilement à l'aide du portique de la centrale. Cette opération s'effectue après assèchement du conduit fermé à l'aval par une vanne et à l'amont par un batardeau d'un type développé spécialement dans le but de reduire l'importance des ouvrages de génie civil.

Les machines, de puissances identiques, munies d'alternateurs à attaque directe comporteraient toujours des turbines d'un diamètre de $8 \mathrm{~m}$ qui pourraient tourner au synchronisme à $51,4 \mathrm{tr} / \mathrm{min}$. La carcasse de l'alternateur, d'un diamètre extérieur de $10 \mathrm{~m}$ et d'une longueur de $2,70 \mathrm{~m}$, comprendrait des ailettes externes de refroidissement. Le diamètre hors tout de la carcasse atteindrait alors $10,60 \mathrm{~m}$. Les conditions de transport imposent une réalisation en plusieurs éléments assemblés sur le site. L'empilage du circuit magnétique et le bobinage du stator doivent s'effectuer sur la plage de montage de la centrale. Le diamètre 
et la longueur du cône intérieur de l'avant-distributeur, raccordé sur la caracasse de l'alternateur, augmentent considérablement. Le rotor d'un diamètre extérieur de $8,64 \mathrm{~m}$ pour une longueur de fer de $0,60 \mathrm{~m}$ affecte la forme d'un disque très plat. Pour accéder au compartiment amont de l'alternateur, où se trouvent les groupes moto-ventilateurs et les alvéoles d'alimentation en huile du servo-moteur des pales de la roue, il faut prévoir un puits vertical reliant l'ogive à une galerie d'accès placée sous le pertuis du groupe. Cette contrainte conduit à un approfondissement local des fouilles, déjà sensiblement accrues par les dimensions de l'alternateur.

L'augmentation des dimensions de l'avant-distributeur se répercutera sur le prix de cet organe. Enfin, le diamètre important de l'alternateur risque d'entraîner un élargissement du pertuis d'entrée du groupe avec pour conséquence un accroissement de l'entr'axe des machines.

\section{Installations marémotrices}

La figure 15 concerne l'un des projets d'aménagement de la centrale marémotrice de Garolim en Corée. L'équipement envisagé comporterait 24 groupes d'une puissance de $20 \mathrm{MW}$. Les turbines, d'un diamètre de $8 \mathrm{~m}$, tourneraient à $50 \mathrm{tr} / \mathrm{min}$ en entraînant à $300 \mathrm{tr} / \mathrm{min}$, par l'intermédiaire d'un multiplicateur de rapport 6 . un alternateur.

L'implantation des machines en chambre ouverte autorise, grâce à la suppression du conduit amont entourant le bulbe, une réduction notable de la longueur de la centrale. Cette disposition d'ensemble se trouve facilitée par l'absence de grilles de protection.

L'isolement d'un groupe s'effectue au moyen d'une vanne placée dans l'aspirateur à l'aval et d'un batardeau constitué d'éléments semi-cylindriques appuyés sur le parement amont du barrage. Un portique de manuten- tion opère la mise en place de ce batardeau qui peut comporter des engins de démontage de l'alternateur et du multiplicateur. Plusieurs dispositions originales peuvent être adoptées pour la conception de cet organe d'isolement.

Le démontage des composants de la turbine se fait au travers du puits existant au droit de la roue.

\section{CONCLUSIONS}

Les recherches sur l'amélioration de la rentabilité globale des aménagements de basses chutes se poursuivent depuis plusieurs décades; elles concernent les groupes verticaux conventionnels du type Kaplan et les groupes axiaux.

Les développements des méthodes de calcul dans les domaines de la mécanique des fluides des turbomachines et de la mécanique des structures conduisent à des progrès qui bénéficient aux deux types de turbines. La première partie de cette étude illustre, par quelques exemples, les applications de ces programmes de calculs.

L'expérience acquise par la construction de plusieurs centaines de groupes bulbes comportant des roues allant de 5 à près de $8 \mathrm{~m}$ de diamètre et l'excellent comportement de ces machines en exploitation confirment que la technologie et les performances de ce matériel se comparent favorablement à celles des turbines Kaplan. Seules, les conditions spécifiques aux projets examinés gouvernent le choix des dimensions qui ne dépendent pratiquement plus de limites constructives. La rentabilité des installations caractérisées par des débits d'équipement importants et des chutes allant de 8 à 15 ou $20 \mathrm{~m}$
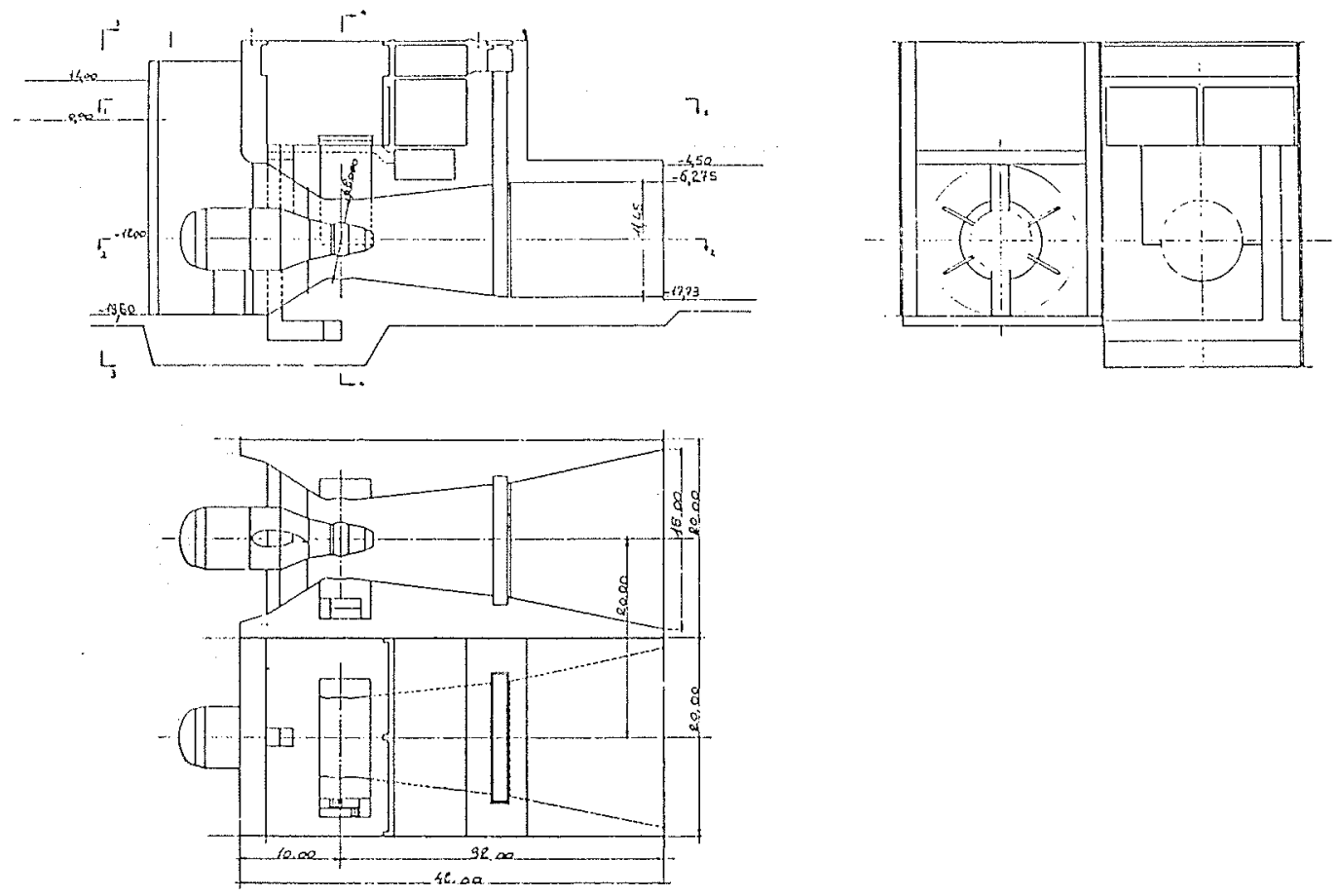

Figure 15. - Centrale marémotrice de Garolim (Corée) - Implantation. 
s'est trouvée très sensiblement améliorée par l'emploi de groupes axiaux du type bulbe amont. Les puissances unitaires des groupes en exploitation depuis des années atteignent $54 \mathrm{MW}$; des projets très avancés prévoient des machines de $64 \mathrm{MW}$, actuellement des groupes de 70 à $100 \mathrm{MW}$ peuvent être envisagés.

La rentabilité de l'équipement des grands fleuves pour des chutes inférieures à $8 \mathrm{~m}$ pose des problèmes et oblige maîtres d'œuvre et constructeurs à rechercher des dispositions d'ensemble mieux adaptées à ce genre d'installations. Les progrès réalisés dans la construction des multiplicateurs de vitesse et l'amélioration considérable de leur fiabilité rendent possible l'utilisation de ces appareils pour l'entrainement d'alternateurs rapides de conception plus traditionnelle mais toujours prévus pour résister à l'emballentent maximal. L'emploi de ces organes, d'un encombrement limité, qui se logent sans difficulté dans un bulbe amont de diamètre réduit, amène les constructeurs à de nouvelles réflexions sur la conception du matériel électromécanique et des ouvrages de génie civil.

Maîtres d'œuvre et constructeurs cherchent toujours à bénéficier, de tout progrès des techniques, sans courir de risque déraisonnable; les quelques voie survolées dans cette étude apportent une contribution à cés progrès vers un meilleur service au meilleur coût.

\section{Discussion}

Président : M. CORDELLE

Le Président remercie M. BOSC pour les indications fournics sur le développement des groupes de basses chutes. De dimensions modestes à leur début, ils peuvent atteindre maintenant des dimensions importantes aussi bien en solution verticale (turbines Kaplan) qu'en solution horizontale (turbines bulbes).

M. HOEPPFNER pose une question au sujet de la réduction du coût qui peut être escomptée lorsque la machine ne comporte qu'un dispositif de réglage simplifié.

$M$. BOSC cite des ordres de grandeur.

En prenant pour dénominateur le coût global de la turbine bulbe avec distributeur mobile et pales mobiles et pour numérateur la différence de coût entre organes mobiles et organes fixes, on obtient un rapport qui vaut sensiblement :

- $25 \%$ pour le distributeur

- 7 \% pour les pales.

M. BOSC signale que s'il vient de présenter l'aspect technique des groupes simplifiés avec distributeur fixe et pales réglables, c'est parce que le fonctionnement à charge partielle de telles machines ne pose pas de problèmes particuliers, ce qui n'est pas le cas des machines à pales non réglables et distributeur mobile. On voit que les appréciations technique et économique vont dans le même sens.

M. ANDRE s'intéresse aux multiplicateurs de vitesse. Il désire connaître les puissances des plus gros multiplicateurs en service industriel et les ordres de grandeur des rendements des multiplicateurs récents.

$M . B O S C$ répond à M. ANDRE.

Réponse à la première question : Neyrpic est en relation avec un certain nombre de constructeurs de multiplicateurs : Cit-Alcatel, Stal-Laval, Citroën.

Selon Stal-Laval, les Suédois ont utilisé des multiplicateurs surdesturbines d'une puissance de 14,3 MW qui sont en cours de démarrage ; un multiplicateur mis en service a bien supporté les anomalies de couplage sur l'alternateur.

La préoccupation actuelle de Neypric est de déterminer une gamme de multiplicateurs en s'appuyant sur un certain nombre de projets concrets. En principe, la puissance admise par ce matériel va jusqu'à $40 \mathrm{MW}$. L'expérience acquise à ce jour se réfère surtout à des puissances de 3-5 MW et pour des cas particuliers jusqu'à $15 \mathrm{MW}$. Neyrpic s'est attaché à travailler avec plusieurs constructeurs ; c'est le souci de fiabilité qui entre en ligne de compte.

Réponse à la deuxième question : Pour un multiplicateur à un étage, on peut s'attendre à un rendement de $99,3 \%$. Pour un multiplicateur à 2 étages un rendement de $98,7 \%$. C'est un ordre de grandeur. Il faut remarquer que cette légère perte de rendement introduite par le multiplicateur est largement compensée par le gain sur l'alternateur dû à sa vitesse plus élevée.

M. COURIER désire connaître les raisons qui ont orienté le choix des réalisations récentes à l'étranger, soit la disposition en chambre d'eau, soit la disposition en conduit et s'il y a une tendance générale en faveur de l'une ou l'autre solution.

$M . B O S C$ répond que cela correspond surtout aux préoccupations du génie civil. La disposition en chambre d'eau permet de déconnecter le montage de la turbine et celui de l'alternateur. On peut faire simultanément le démontage et l'entretien de la turbine et de l'alternateur, et les commodités d'accès sont grandement facilitées.

A l'origine, on était surtout préoccupé par l'accessibilité à la turbine. En fait, c'est l'alternateur qui a posé le plus de problèmes. M. BOSC se souvient d'une réunion tenue, il y a deux ou trois ans, à la Société Hydrotechnique de France, où avait été évoqué l'aspect du démontage de l'alternateur indépendamment de celui de la turbine. La disposition en chambre d'eau avait devancé ce désir.

M. MEIER demande s'il $\mathrm{y}$ a des raisons particulières à ne pas utiliser de servo-moteurs individucls sur les distributeurs des turbines Kaplan comme cela est pratiqué sur les turbines Francis vues précédemment. Les dimensions des distributeurs paraissant les mêmes.

$M . B O S C$ répond qu'il n'y a aucune raison spéciale. Dans l'un des exposés précédents, les turbines Kaplan de Tchi Li Long en Chine ont été citces. Le diamètre de la roue est de $8 \mathrm{~m}$; la puissance unitaire de $75 \mathrm{MW}$ sous $22 \mathrm{~m}$ (puissance limitée). Les directrices sont munies de servo-moteurs individuels synchronisés mécaniquement. Pour certains groupes du Rhône, on a utilisé des servo-moteurs individuels avec synchronisation électrique.

Le choix entre commandes des directrices par cercle de vannage ou par servo-moteurs indépendants dépend des considérations économiques et du degré de sécurité recherché pour la fermeture di distributeur (en cas de grippage de servo-moteurs).

Par exemple, les turbines de Palmar (diamètre de roue $7,60 \mathrm{~m}$, puissance $113,4 \mathrm{MW}$ sous $27,15 \mathrm{~m}$ ) ont leur distributeur commandé par un cercle de vannage mû par quatre servomoteurs attachés sur le flasque support du pivot. Une pompe spéciale haute pression ( 80 bars) assure la fermeture des pales en cas de survitesse de la machine.

En revanche, pour les turbines de Tchi Li Long, comme il n'y a pas de dispositif de fermeture des pales à l'emballement, 
c'est le distributeur qui assure cette manœuvre et la solution avec servo-moteurs individuels a été retenues.

La même remarque peut être faite pour les turbines-pompes. Des turbines-pompes Neyrpic ont l'un ou l'autre des systèmes.

Si l'on maîtrise correctement les divers jeux, la commande par cercle de vannage a beaucoup d'arguments en sa faveur car elle est plus économique. Le seul risque est un grippage de servo-moteurs.

M. HAMON demande à M. BOSC quelle est son opinion sur le groupe bulbe à alternateur périphérique dont il a été parlé la veille.

M. BOSC pense qu'on pourrait entendre le représentant de la Société Escher-Wyss pour discuter de cette question.

Il fait une remarque préliminaire. Les constructeurs soviétiques ont fait l'expérience d'alternateur périphérique de grande puissance ; ils ont abandonne cette technique. Il est evident qu'un alternateur périphérique conduit à ajouter une masse importante à un organe (la roue) de dimensions plus ou moins grandes : si les pales de la roue ne sont pas réglables, cela peut se concevoir ; mais si les pales de la roue sont réglables, les difficultés s'accroissent notablement. Comment déterminer le comportement vibratoire d'un tel ensemble ? Comment être sûr de la fiabilité des 2 joints de grand diamètre ? On sait bien que les joints sont les "bêtes à chagrin" des exploitants ; l'augmentation de diamètre n'est certainement pas de nature à diminuer les risques d'ennuis.

M. le Président qui ne désire pas ouvrir une discussion de spécialistes sur cette question, demande au représentant d'EscherWyss s'il souhaite prendre la parole.
M. MEIER rappelle que les problèmes essentiels ont été mentionnés dans l'exemple montré à la séance de la veille. Escher-Wyss a construit une turbine avec pales réglables d'un diamètre de roue $3,70 \mathrm{~m}$ et fabrique actuellement une turbine avec pales non réglables d'un diamètre de 7,60 m. Escher Wyss pense avoir résolu le problème d'étanchéité sur l'anneau de la rove.

Les avantages de la conception Straflo comparée aux turbines bulbes sont en principe :

- économie dans les ouvrages de génie civil par une unité turbine-alternateur très compacte ;

- pas de limite concernant les puissances maximales sans problèmes de refroidissement ;

- alternateurs moins chers

- moment d'inertie plus élevé.

Les désavatanges sont :

- rendements inférieurs comparativement aux turbines bulbes, mais à peu près semblables à ceux d'une turbine Kaplan.

M. MEIER précise qu'Escher-Wyss étudie tout comme Neyrpic les groupes bulbes avec multiplicateurs de grande puissance qui permettent d'obtenir des avantages.

Pour le Président, il est certain que lorsque il y a un besoin nouveau, plusieurs solutions se trouvent en concurrence. L'avenir dira si l'un des systèmes s'impose par rapport à l'autre ou si les deux peuvent se développer parallèlement.

En conclusion, $M . B O S C$ tient tout de même à faire remarquer qu'à son avis la solution avec alternateur périphérique est une solution à haut risque. 